\title{
A spherical model for orientation and spatial-frequency tuning in a cortical hypercolumn
}

\author{
Paul C. Bressloff ${ }^{1}$ and Jack D. Cowan ${ }^{2^{*}}$ \\ ${ }^{1}$ Department of Mathematics, University of Utah, Salt Lake City, UT 84112, USA (bresslof@math.utah.edu) \\ ${ }^{2}$ Mathematics Department, University of Chicago, Chicago, IL 60637, USA
}

\section{CONTENTS}

1. Introduction

Part I: Mean-field theory 1646

2. Details of the spherical model 1646

$\begin{array}{lr}\text { 3. Stationary localized states } & 1647\end{array}$

$\begin{array}{lr}\text { (a) Broad activity profile } & 1648\end{array}$

$\begin{array}{ll}\text { (b) Narrow activity profile } & 1649\end{array}$

$\begin{array}{ll}\text { 4. Orientation and spatial-frequency tuning curves } & 1651\end{array}$

Part II: Receptive fields and cortico-geniculate feedback $\quad 1654$

5. Feed-forward receptive fields 1654

6. Spherical harmonic projection of the LGN input 1656

7. Renormalizing the LGN input 1658

(a) Feed-forward mechanisms 1658

(b) Cortico-geniculate feedback 1659

8. Cross-orientation suppression 1660

9. Discussion 1662

$\begin{array}{ll}\text { Appendix A } & 1664\end{array}$

$\begin{array}{ll}\text { Appendix B } & 1664\end{array}$

References 1665

A theory is presented of the way in which the hypercolumns in primary visual cortex (V1) are organized to detect important features of visual images, namely local orientation and spatial frequency. Given the existence in V1 of dual maps for these features, both organized around orientation pinwheels, we constructed a model of a hypercolumn in which orientation and spatial-frequency preferences are represented by the two angular coordinates of a sphere. The two poles of this sphere are taken to correspond, respectively, to high and low spatial-frequency preferences.

In Part I of the paper, we use mean-field methods to derive exact solutions for localized activity states on the sphere. We show how cortical amplification through recurrent interactions generates a sharply tuned, contrast-invariant population response to both local orientation and local spatial frequency, even in the case of a weakly biased input from the lateral geniculate nucleus (LGN). A major prediction of our model is that this response is non-separable with respect to the local orientation and spatial frequency of a stimulus. That is, orientation tuning is weaker around the pinwheels, and there is a shift in spatialfrequency tuning towards that of the closest pinwheel at non-optimal orientations.

In Part II of the paper, we demonstrate that a simple feed-forward model of spatial-frequency preference, unlike that for orientation preference, does not generate a faithful representation when amplified by recurrent interactions in V1. We then introduce the idea that cortico-geniculate feedback modulates LGN activity to generate a faithful representation, thus providing a new functional interpretation of the role of this feedback pathway. Using linear filter theory, we show that if the feedback from a cortical cell is taken to be approximately equal to the reciprocal of the corresponding feed-forward receptive field (in the two-dimensional Fourier domain), then the mismatch between the feed-forward and cortical frequency representations is eliminated. We therefore predict that cortico-geniculate feedback connections innervate the LGN in a pattern determined by the orientation and spatial-frequency biases of feed-forward receptive fields. Finally, we show how recurrent cortical interactions can generate cross-orientation suppression.

Keywords: orientation; spatial frequency; hypercolumn; neural modelling; cortico-geniculate feedback 


\section{INTRODUCTION}

A prominent feature of the functional architecture of the visual cortex (V1) is the existence of an orderly retinotopic mapping of the visual field onto its surface, with left and right halves of the visual field mapped onto the left and right V1, respectively. Superimposed upon this are additional maps reflecting the fact that neurons respond preferentially to stimuli with particular features such as orientation and ocularity (Hubel \& Wiesel 1977; Obermayer \& Blasdel 1993; Swindale 1996). Maps of both ocularity and orientation preference have been well characterized in cat and monkey, via microelectrode recording (Hubel \& Wiesel 1962, 1968, 1977) autoradiographic studies using proline (Wiesel et al. 1974) or 2deoxyglucose (2-DG) (Hubel et al. 1978), and optical imaging (Blasdel \& Salama 1986; Bonhoeffer \& Grinvald 1991; Blasdel 1992). The topography revealed by these methods has several characteristic features (Obermayer \& Blasdel 1993). (i) Orientation preference changes continuously as a function of cortical location, except at singularities or pinwheels. (ii) There exist linear zones, ca. $750 \mu \mathrm{m} \times 750 \mu \mathrm{m}$ in area (in macaques), bounded by pinwheels, within which iso-orientation regions form parallel slabs. (iii) Linear zones tend to cross the borders of ocular dominance stripes at right angles; pinwheels tend to align with the centres of ocular dominance stripes. All these features can be seen in the optical image shown in figure 1 .

These observations suggest that the microstructure of $\mathrm{V} 1$ is spatially periodic with a period of ca. $1 \mathrm{~mm}$ (in primates). The fundamental domain of this tiling of the cortical plane is the hypercolumn (Hubel \& Wiesel 1974), which contains the full range of orientation preferences $\phi \in[0, \pi]$ organized around pinwheels, with one set of preferences for each ocular dominance column. The identification of the hypercolumn as a basic cortical module is still somewhat controversial (LeVay \& Nelson 1991). However, it has proved a very useful conceptual tool in the development of large-scale dynamic models of cortical function. In its original form, the hypercolumn was organized in terms of linear zones of orientation preference slabs and ocular dominance columns, as shown in figure $2 a$. This was later modified to include the cytochrome oxidase (CO) blobs observed in the macaque by Horton \& Hubel (1981) (see figure $2 b$ ) and only later found in the cat (Murphy et al. 1995). The blobs are regions of cells that are more metabolically active and hence richer in their levels of CO. They tend to be located at the centres of ocular dominance stripes and have a strong association with approximately half the orientation singularities.

The fact that orientation preference is a periodic quantity suggests that the internal structure of a hypercolumn can be idealized as a ring of orientation-selective wedges or patches. In the past decade, several network models have appeared based on such an idealization (Ben-Yishai et al. 1995, 1997; Somers et al. 1995, 1998; Vidyasagar et al. 1996; Mundel et al. 1997; Li 1999; Bressloff et al. 2000; Dragoi \& Sur 2000; Stetter et al. 2000; Bressloff \& Cowan $2002 a$ ). These models have been used to investigate the role of intra-cortical interactions in orientation selectivity and tuning. The classical model of Hubel \& Wiesel (1962) proposes that the orientation preference of

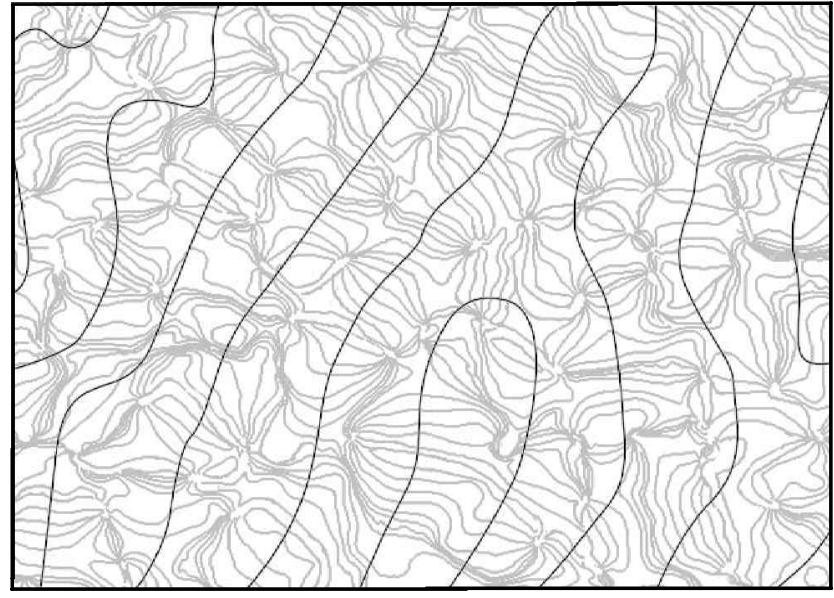

Figure 1. Iso-orientation (light) and ocular dominance (dark) contours in a small region of macaque VI (redrawn from Blasdel 1992, with permission).

a cortical neuron arises primarily from the geometric alignment of the receptive fields of thalamic neurons in the lateral geniculate nucleus (LGN) projecting to it. This has been confirmed by several recent experiments (Reid \& Alonso 1995; Ferster et al. 1997). However, there is also growing experimental evidence suggesting the importance of intra-cortical feedback for orientation tuning. For example, the blockage of extracellular inhibition in the cortex leads to considerably broader tuning (Sillito 1975; Nelson et al. 1994). Moreover, intracellular measurements indicate that direct inputs from the LGN to neurons in layer 4 of the visual cortex provide only a fraction of the total excitatory inputs relevant to orientation selectivity (Douglas et al. 1995). Several modelling studies have shown how local recurrent interactions within an isolated cortical hypercolumn (idealized as a ring network) can amplify certain Fourier components of network activity leading to sharp orientation tuning curves, even when the LGN inputs are weakly biased (Ben-Yishai et al. 1995, 1997; Somers et al. 1995; Bressloff et al. 2000). Such an amplification mechanism provides one possible explanation for the approximate contrast invariance of the tuned response. Subsequently, more large-scale models of a cortex, based on a system of coupled ring networks, have been used to investigate how orientation tuning is modulated by long-range interactions between hypercolumns (Mundel et al. 1997; Somers et al. 1998; Li 1999; Dragoi \& Sur 2000; Stetter et al. 2000; Bressloff \& Cowan 2002a).

Although ring models have been quite successful in accounting for some aspects of the response properties of hypercolumns, they have several limitations. For example, they do not take into account the two-dimensional structure illustrated in figure 1 , in which iso-orientation pinwheels alternate with linear zones, nor the presence of ocular dominance columns. More significantly, for our interest, they also neglect the spatial frequency selectivity of V1 neurons. Such selectivity has been observed in many physiological experiments. Recordings from cat and monkey striate cortex have established that a large number of cells are narrowly tuned to spatial frequency. Figure 3, for example, shows the responses of several macaque monkey V1 cells to oriented gratings. The average bandwidth is 
(a)

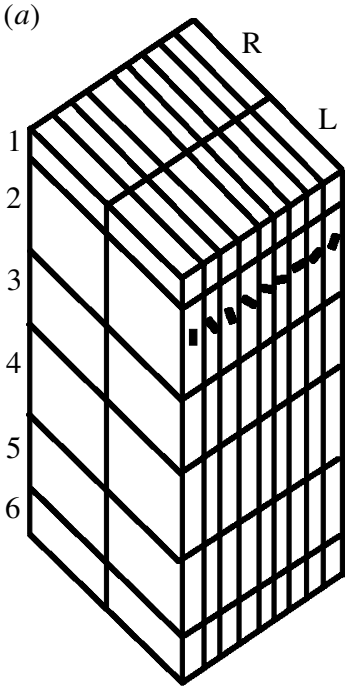

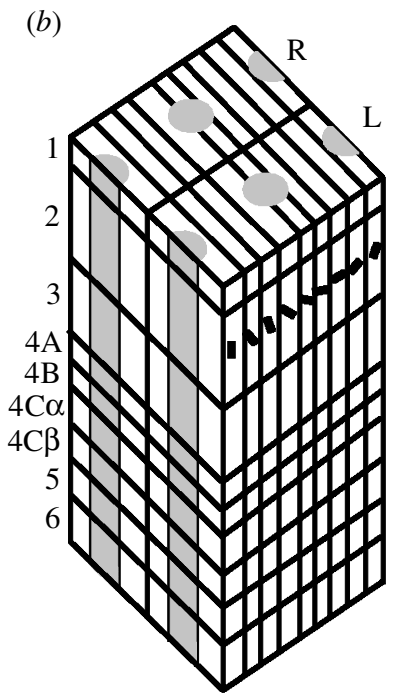

(b)

Figure 2. (a) Hubel and Wiesel's original icecube model of a V1 hypercolumn, redrawn for the cat. (b) The icecube model with CO blobs for macaque V1.

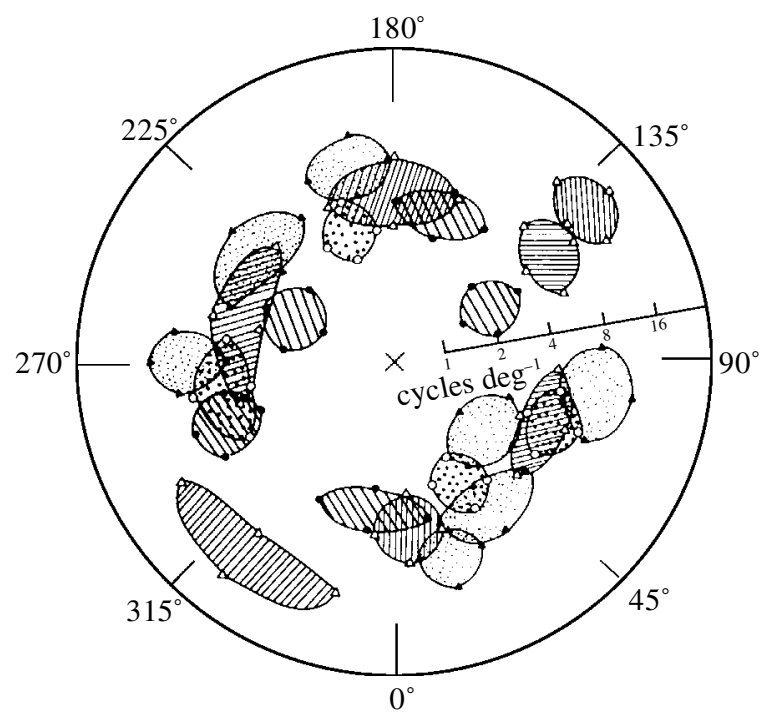

Figure 3. Spatial frequency and orientation selectivity of cells in macaque V1. The thresholded response of several cells is plotted as a function of stimulus spatial frequency and orientation. The results are shown in log-polar coordinates with orientation given by the polar angle and spatial frequency by the radius (on a logarithmic scale) (redrawn from De Valois et al. 1982, with permission).

between one and two octaves, which covers a small fraction of the total range of spatial frequencies (approximately six to eight octaves in the fovea) to which the macaque is sensitive (De Valois \& De Valois 1988). As in the case of psychophysical studies (Kelly \& Magnuski 1975), two-dimensional stimuli, such as checkerboards, provide strong evidence that neurons are tuned to two-dimensional spatial frequencies. In fact, there is considerable physiological evidence to suggest that cortical neurons act like band-pass filters for both orientation and spatial frequency, so that a hypercolumn implements a localized or windowed two-dimensional spatial-frequency filtering of a stimulus, rather than simply performing local edge detection (Webster \& De Valois 1985; Jones \& Palmer 1987).

The distribution of spatial-frequency preference across the cortex is less clear than that of orientation preference. Nevertheless, based on the 2-DG studies available at the time (see Tootell et al. 1981), De Valois \& De Valois (1988) introduced the models of V1 hypercolumns shown in figure 4 . In the macaque, it was found that the $\mathrm{CO}$ blob regions were sites of cells that responded preferentially to low spatial frequencies, which suggested that spatial frequency increased radially, away from the blobs. This impression has recently been extended by optical studies of the spatial-frequency map in the cat (Bonhoeffer et al. 1995; Hübener et al. 1997; Issa et al. 2000). These studies indicate that: (i) both orientation and spatial-frequency preferences are distributed almost continuously across cortex; (ii) spatial-frequency preferences at both extremes of the continuum tend to be located at orientation pinwheels (i.e. the pinwheels that do not coincide with $\mathrm{CO}$ blobs correspond to regions of high spatial frequency); and (iii) around the pinwheels iso-orientation and iso-frequency preference contours are approximately orthogonal (see figure 5). Note that in most local neighbourhoods of the region of V1 shown in figure 5 one can identify a low and a high spatial frequency pinwheel connected by a linear zone. In a few cases, high spatial frequency pinwheels are connected by linear zones. However, they tend to be sited in different ocular dominance columns.

Motivated by such considerations, we introduce a minimal model of a hypercolumn that: (i) includes both orientation and spatial frequency preferences; (ii) incorporates the orientation preference pinwheels; and (iii) exhibits sharply tuned responses in the presence of recurrent interactions and weakly biased LGN inputs. For simplicity, we restrict ourselves to a single ocular dominance column and a single cortical layer. In the ring model of orientation tuning the synaptic weights are taken to depend on the difference between the orientation preference of pre- and post-synaptic neurons, which naturally leads to a ring or circular network topology. Given that spatial frequency is not a periodic variable within a hypercolumn, we cannot extend the ring model by including a second ring so that the network topology becomes a torus. The simplest choice is to assume the topology is a cylinder, as shown in figure 6. This leads to a network response that is separable with respect to the two stimulus features. However, recent experimental results suggest that although separability appears to hold in the linear zones of the orientation map, there is significant non-separability close to the orientation pinwheels (Maldonado et al. 1997; Issa et al. 2000; Mazer et al. 2002). Combining this with the assumption that each hypercolumn typically contains two orientation pinwheels per ocular dominance column, and that these correspond respectively to the two extremes of spatial frequency within the hypercolumn, we introduce the network topology of a sphere to model a hypercolumn, with its two pinwheels identified as the north and south poles, respectively (see figure 7).

It is important to distinguish between the network topology shown in figure 6 or 7 , which deals with synaptic weights as a function of orientation and spatial-frequency preference labels, and the actual two-dimensional spatial arrangement of neurons within a single cortical layer (see 
(a)

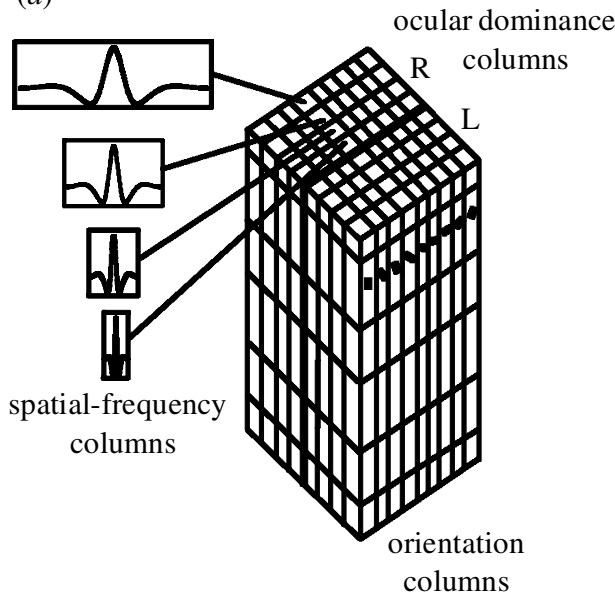

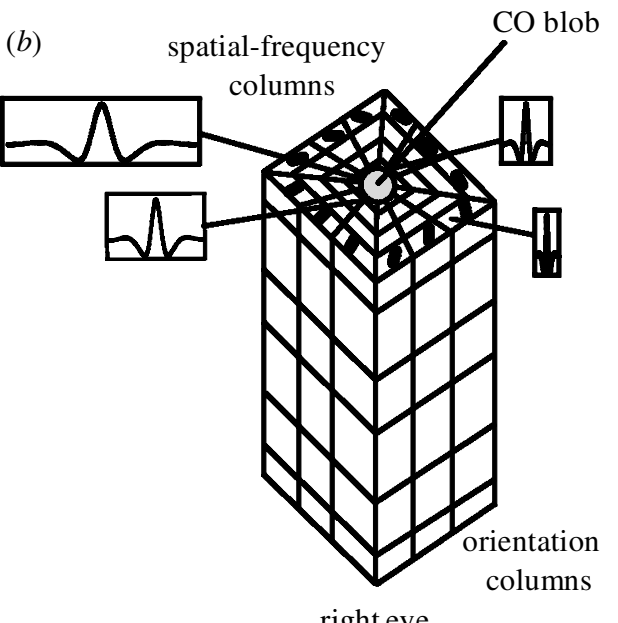

right eye

Figure 4. (a) De Valois and De Valois' modified icecube model of a cat V1 hypercolumn. (b) The modified icecube model with CO blobs for macaque V1 (redrawn from De Valois \& De Valois 1988, with permission).

figure 4). As in the ring model, the spherical model of a hypercolumn is an abstraction from a complicated set of experimental results such as those presented in figures 1 and 5. The model does not account for all of the details apparent in these figures. In fact, it should also be noted that optical imaging data are inherently noisy so that some of the conclusions regarding the spatial frequency map and the nature of orientation pinwheels are still quite controversial. Nevertheless, we believe that the analysis of conceptual models such as the one presented in this paper can lead to insights into the true nature of the action of V1.

\section{PART I: MEAN-FIELD THEORY}

In Part I, we present a dynamic theory of orientation and spatial-frequency tuning in a cortical hypercolumn whose network topology is taken to be spherical. As we have already indicated in $\mathbb{} 1$, this topology naturally accommodates the two orientation preference pinwheels (within a single ocular dominance column), which are located at the poles of the sphere, as well as the twodimensional curvilinear coordinate system we choose to represent orientation and spatial-frequency preferences within a hypercolumn. Explicit solutions for localized activity states on the sphere are obtained using a meanfield approach (Ben-Yishai et al. 1995; Hansel \& Sompolinsky 1997). We thus show how cortical amplification through recurrent interactions generates a sharply tuned, contrast-invariant population response to both orientation and spatial frequency. A major prediction of our model is that this response is non-separable with respect to these stimulus features due to the presence of the pinwheels. (A preliminary version of the spherical model has been reported briefly elsewhere (Bressloff \& Cowan 2002b). In particular, we used a perturbative amplitude equation approach to establish the basic principle of cortical amplification via spontaneous symmetry breaking. However, our analysis was restricted to the weakly nonlinear regime. Here, we greatly extend the analysis using the meanfield approach.)

\section{DETAILS OF THE SPHERICAL MODEL}

We assume that a hypercolumn is parametrized by two cortical labels, which represent the orientation preference $\phi \in[0, \pi)$ and spatial-frequency preference $p \in\left[p_{\min }, p_{\text {max }}\right]$ of a local patch or column of cells. Typically, the bandwidth of a hypercolumn is between three and four octaves, that is, $p_{\max } \approx 2^{n} p_{\text {min }}$ with $n=4$. This is consistent with the observations of Hubel \& Wiesel (1974), who found a twooctave scatter of receptive field sizes at each cortical region they mapped. Motivated by the optical imaging data described in $\int 1$, we assume that the network topology is a sphere $S^{2}$ with the two pinwheels identified as the north and south poles, respectively (see figure 7). If we take $(\theta, \phi)$ to be the angular coordinates on the sphere with $\theta \in[0, \pi), \phi \in[0, \pi)$ then $\theta$ determines the spatial-frequency preference $p$ according to

$\theta \equiv \mathcal{L}(p)=\pi \frac{\log \left(p / p_{\mathrm{min}}\right)}{\log \left(p_{\mathrm{max}} / p_{\mathrm{min}}\right)}$.

That is, $\theta$ varies linearly with $\log p$. This is consistent with experimental data that suggest a linear variation of $\log p$ with cortical separation (Issa et al. 2000). This leads to the spherical coordinate system shown in figure 8 .

Let $a(\theta, \phi, t)$ denote the activity of a local population of cells on the sphere with angular coordinates $(\theta, \phi)$. The evolution equation for the state $a(\theta, \phi, t)$ is taken to be of the form

$\frac{\partial a(\theta, \phi, t)}{\partial t}=-a(\theta, \phi, t)+[I(\theta, \phi, t)-\kappa]_{+}$,

where $\kappa$ is a threshold and $I(\theta, \phi, t)$ is the total synaptic current,

$I(\theta, \phi, t)=\int_{s^{2}} w\left(\theta, \phi \mid \theta, \phi^{\prime}\right) a\left(\theta^{\prime}, \phi^{\prime}, t\right) \mathscr{D}\left(\theta^{\prime}, \phi^{\prime}\right)+h(\theta, \phi)$

with $\mathscr{D}(\theta, \phi)=\sin \theta \mathrm{d} \theta \mathrm{d} \phi / 2 \pi$ the integration measure on the sphere. Here $w$ represents the distribution of recurrent interactions within the hypercolumn and $h(\theta, \phi)$ is a weakly biased input from the LGN. Equation (2.2) is the 
natural extension of the activity-based ring model of orientation tuning considered by Ben-Yishai et al. (1995, 1997). To generalize the amplification mechanism of the ring model to the spherical model (equation (2.2)), we first construct a weight distribution that is invariant with respect to coordinate rotations and reflections of the sphere, that is, the symmetry group $O(3)$. This spherical symmetry, which generalizes the $O(2)$ circular symmetry of the ring model, implies that the pattern of connections within the hypercolumn depends only on the relative distance of cells on the sphere as determined by their angular separation along geodesics or great circles. That is, given two points on the sphere $(\theta, \phi)$ and $\left(\theta^{\prime}, \phi^{\prime}\right)$ their angular separation $\alpha$ is (see figure 8 )

$\cos \alpha=\cos \theta \cos \theta^{\prime}+\sin \theta \sin \theta^{\prime} \cos \left(2\left[\phi-\phi^{\prime}\right]\right)$.

This suggests that the simplest non-trivial form for the weight distribution $w$ is

$w\left(\theta, \phi \mid \theta^{\prime}, \phi^{\prime}\right)=W_{0}+W_{1}\left(\cos \theta \cos \theta^{\prime}\right.$

$$
\left.+\sin \theta \sin \theta^{\prime} \cos \left(2\left[\phi-\phi^{\prime}\right]\right)\right) \text {. }
$$

In figure 9, we plot $w$ as a function of $(\theta, \phi)$ for $\theta^{\prime}=\theta$, $\phi^{\prime}=0$ and $W_{1}>W_{0}$. It can be seen that away from the pinwheels (poles of the sphere at $\theta=0, \pi$ ), cells with similar orientation excite each other whereas those with differing orientation inhibit each other. This is the standard interaction assumption of the ring model (Ben-Yishai et al. 1995; Somers et al. 1995), which has recently received experimental support (Roerig \& Chen 2002). However, around the pinwheels, all orientations uniformly excite, which is consistent with the fact that although the cells around a pinwheel can differ greatly in their orientation preference, they are physically close together within the hypercolumn.

It is possible to construct a more general form of $O(3)$ invariant weight distribution using spherical harmonics. Any sufficiently smooth function $a(\theta, \phi)$ on the sphere can be expanded in a uniformly convergent double series of spherical harmonics

$a(\theta, \phi)=\sum_{n=0}^{\infty} \sum_{m=-n}^{n} a_{n m} Y_{n}^{m}(\theta, \phi)$.

The functions $Y_{n}^{m}(\theta, \phi)$ constitute the angular part of the solutions of Laplace's equation in three dimensions, and thus form a complete orthonormal set. The orthogonality relation is

$\int_{S^{2}} Y_{n_{1}}^{m_{1} *}(\theta, \phi) Y_{n_{2}}^{m_{2}}(\theta, \phi) \mathscr{D}(\theta, \phi)=\frac{1}{4 \pi} \delta_{n_{1}, n_{2}} \delta_{m_{1}, m_{2}}$.

The spherical harmonics are given explicitly by

$Y_{n}^{m}(\theta, \phi)=(-1)^{m} \sqrt{\frac{(2 n+1)(n-m) !}{4 \pi(n+m) !}} P_{n}^{m}(\cos \theta) \mathrm{e}^{2 i m \phi}$

for $n \geqslant 0$ and $-n \leqslant m \leqslant n$, where $P_{n}^{m}(\cos \theta)$ is an associated Legendre function. (Note that we have adjusted the definition of the spherical harmonics to take into account the fact that $\phi$ takes values between 0 and $\pi$.) The action of $S O(3)$ on $Y_{n}^{m}(\theta, \phi)$ involves $(2 n+1) \times(2 n+1)$ unitary matrices associated with irreducible representations of $S U(2)$ (Arfken 1985). From the unitarity of these rep- resentations, one can construct an $O(3)$ invariant weight distribution of the general form

$w\left(\theta, \phi \mid \theta^{\prime}, \phi^{\prime}\right)=4 \pi \sum_{n=0}^{\infty} W_{n} \sum_{m=-n}^{n} Y_{n}^{m^{*}}(\theta, \phi) Y_{n}^{m}\left(\theta^{\prime}, \phi^{\prime}\right)$

with $W_{n}$ real. For simplicity, we shall neglect higher harmonic contributions to $w$ by setting $W_{n}=0$ for $n \geqslant 2$ so that equation (2.9) reduces to equation (2.5) on rescaling $W_{1}$.

Finally, the weakly biased LGN input $h(\theta, \phi)$ is assumed to be of the form

$$
\begin{aligned}
h(\theta, \phi)= & C[1-\varepsilon+\varepsilon(\cos \Theta \cos \theta \\
& +\sin \Theta \sin \theta \cos (2[\phi-\Phi]))] .
\end{aligned}
$$

This represents a unimodal function on the sphere with a single peak at $(\Theta, \Phi)$. Here, $C$ is the effective contrast of the input and $\varepsilon$ measures the degree of bias. In fact, equation (2.10) is the projection of the feed-forward input from the LGN onto the zeroth and first order spherical harmonics. The a posteriori justification for this is based on the idea that recurrent interactions within the hypercolumn amplify these particular components of the feed-forward input, therefore higher order harmonics can be neglected (Bressloff \& Cowan 2002b). We also note that recent optical imaging experiments provide strong support for the role of recurrent interactions in cortical amplification (Sharon \& Grinvald 2002). Rectification arising from the firing rate characteristics of cortical cells then leads to a sharply tuned, contrast-invariant response to both orientation and spatial frequency (see $\ 3$ ). The peak response, which is located at $(\Theta, \Phi)$, is assumed to faithfully encode the spatial frequency $p_{\mathrm{s}}$ and orientation $\phi_{\mathrm{s}}$ of an external visual stimulus, that is, $\Theta=2\left(p_{\mathrm{s}}\right)$ and $\Phi=\phi_{\mathrm{s}}$. However, as we discuss in Part II, the relationship between $\Theta$ and $p_{\mathrm{s}}$ is far from straightforward. The transformation from visual stimulus to cortical input is typically described in terms of a convolution with respect to a feedforward receptive field modelled, for example, as a difference of Gaussians (Hawken \& Parker 1987). If the loworder spherical harmonic components of the resulting feed-forward input to a hypercolumn are now amplified, one finds that the cortical spatial frequency is shifted relative to the stimulus frequency - there is no corresponding shift in orientation. In other words, the network does not faithfully encode the stimulus spatial frequency unless an additional filtering operation is introduced. We suggest, in Part II, that feedback from V1 back to LGN (Murphy et al. 1999) can modulate LGN activity to produce a faithful encoding of spatial frequency. However, we ignore these subtleties here and proceed with the form of LGN input given by equation (2.10).

\section{STATIONARY LOCALIZED STATES}

It is convenient to introduce real versions of the firstorder harmonics,

$f_{0}(\theta, \phi)=\cos \theta$

$f_{+}(\theta, \phi)=\sin \theta \cos 2 \phi, f_{-}(\theta, \phi)=\sin \theta \sin 2 \phi$,

so that equations (2.5) and (2.10) can be rewritten in the form 


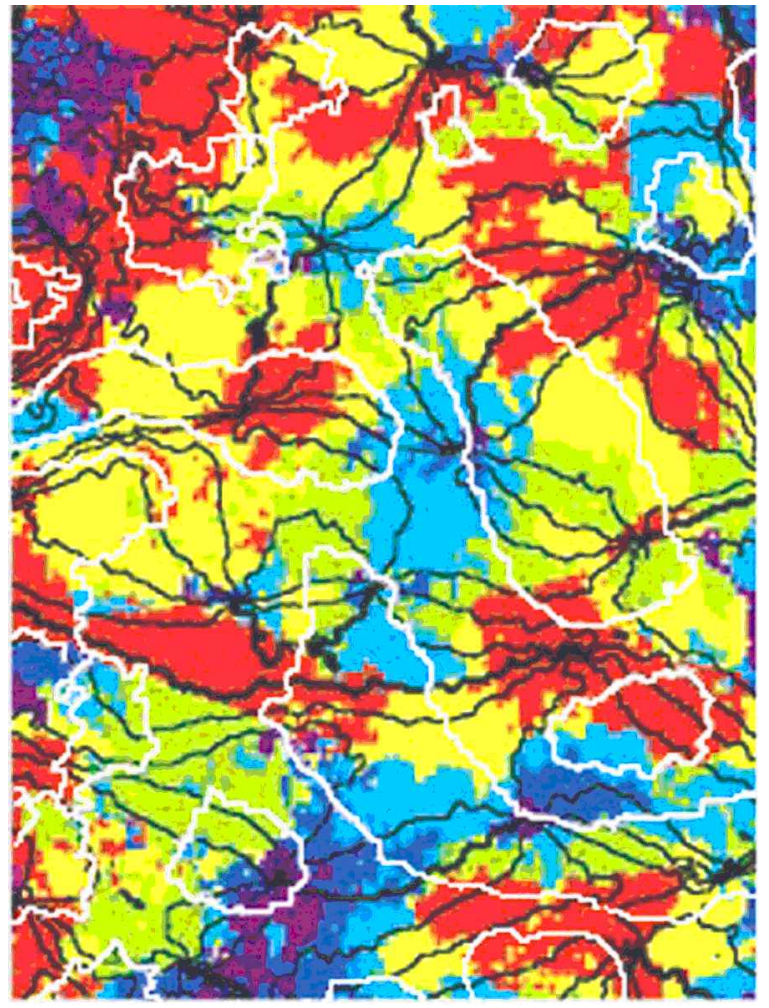

Figure 5. Map of iso-orientation preference contours (black lines), ocular dominance boundaries (white lines), and spatial frequency preferences of cells in the cat V1 (redrawn from Issa et al. 2000, with permission). Red regions correspond to low spatial-frequency preference, violet to high.

$$
w\left(\theta, \phi \mid \theta^{\prime}, \phi^{\prime}\right)=W_{0}+W_{1} \sum_{m=0, \pm} f_{m}(\theta, \phi) f_{m}\left(\theta^{\prime}, \phi^{\prime}\right)
$$

and

$h(\theta, \phi)=\left[1-\varepsilon+\varepsilon \sum_{m=0, \pm} f_{m}(\Theta, \Phi) f_{m}(\theta, \phi)\right]$,

with

$$
\sum_{m=0, \pm} f_{m}(\theta, \phi) f_{m}\left(\theta^{\prime}, \phi^{\prime}\right)
$$

equal to the angular separation of $(\theta, \phi)$ from $\left(\theta^{\prime}, \phi^{\prime}\right)$. Substituting equations (2.3), (3.2) and (3.3) into the evolution equation (2.2) then gives

$$
\begin{aligned}
\frac{\partial a(\theta, \phi, t)}{\partial t}= & -a(\theta, \phi, t) \\
& +\left[I_{0}(t)+\sum_{m=0, \pm} I_{1}^{m}(t) f_{m}(\theta, \phi)\right]_{+},
\end{aligned}
$$

where

$I_{0}(t)=C(1-\varepsilon)+W_{0} R_{0}(t)-\kappa$,

$I_{1}^{m}(t)=C \varepsilon f_{m}(\Theta, \Phi)+W_{1} R_{1}^{m}(t)$

and $R_{0}, R_{1}^{m}$ are the order parameters

$R_{0}(t)=\int_{s^{2}} a(\theta, \phi, t) \mathscr{D}(\theta, \phi)$,

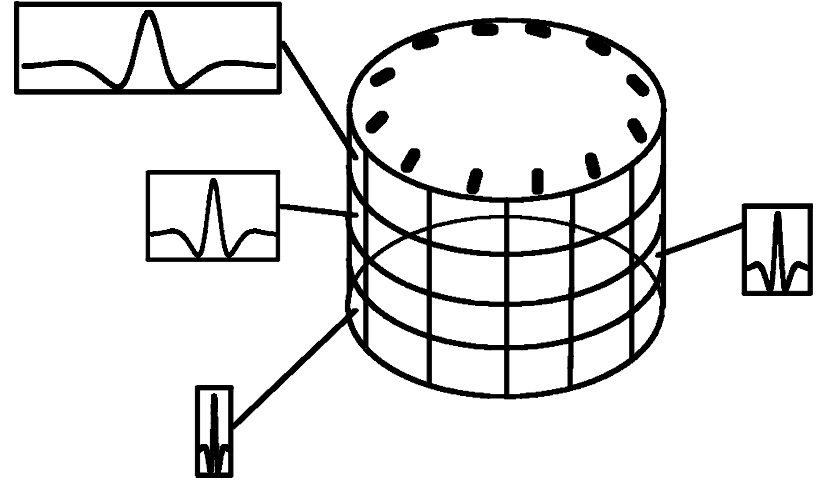

Figure 6. A cylindrical network topology. Spatial-frequency preference decreases from top to bottom whereas orientation preference varies around the circumference of the cylinder.

$R_{1}^{m}(t)=\int_{s^{2}} a(\theta, \phi, t) f_{m}(\theta, \phi) \mathscr{D}(\theta, \phi)$.

Following along similar lines to the analysis of the ring model (Ben-Yishai et al. 1995; Hansel \& Sompolinsky 1997), we studied fixed-point solutions of equation (3.4) in which the activity surface is centred at the peak of the LGN input $(\Theta, \Phi)$. That is,

$a(\theta, \phi)=\left[I_{0}+\sum_{m=0, \pm} I_{1}^{m} f_{m}(\theta, \phi)\right]_{+}$.

Such a solution is self-consistent provided that at the fixed point $R_{1}^{m}=R_{1} f_{m}(\Theta, \Phi)$ for some $R_{1}$. Given such a fixed-point solution, we define the network gain $G$ as the ratio between the maximal activity and the contrast relative to threshold

$G=\frac{a(\Theta, \Phi)}{C-\kappa}$.

It is useful to distinguish between broad and narrow activity profiles $a(\theta, \phi)$. We say that the profile is broad when all the cells are above threshold. That is, $I(\theta, \phi)$ $\geqslant \kappa$ and hence $a(\theta, \phi)>0$ for all $(\theta, \phi) \in S^{2}$. However, a narrow profile is one for which $a(\theta, \phi)$ is only non-zero over a subdomain $\Sigma=\left\{\theta, \phi \mid 0 \leqslant \theta<\theta_{0}(\phi), 0 \leqslant \phi<\pi\right\}$ $\subset S^{2}$ : this is what we mean by a localized state. The closed curve $\theta=\theta_{0}(\phi)$ determines the boundary of the localized state on the sphere. Note that although the twodimensional activity profile on the sphere is localized, it is not necessary that the resulting orientation tuning curves should, themselves, be localized (see $₫ 4$ ).

\section{(a) Broad activity profile}

The analysis of a broad activity profile is relatively straightforward, since the fixed point equation (3.9) reduces to

$a(\theta, \phi)=I_{0}+\sum_{m=0, \pm} I_{1}^{m} f_{m}(\theta, \phi)$,

which can be substituted into equations (3.7) and (3.8) to give $R_{0}=I_{0}$ and $R_{1}^{m}=I_{1}^{m} / 3$. It follows from equations (3.5) and (3.6) that

$$
R_{0}=\frac{C(1-\varepsilon)-\kappa}{1-W_{0}}, R_{1}^{m}=R_{1} f_{m}(\Theta, \Phi), R_{1}=\frac{C \varepsilon / 3}{1-W_{1} / 3}
$$

and 


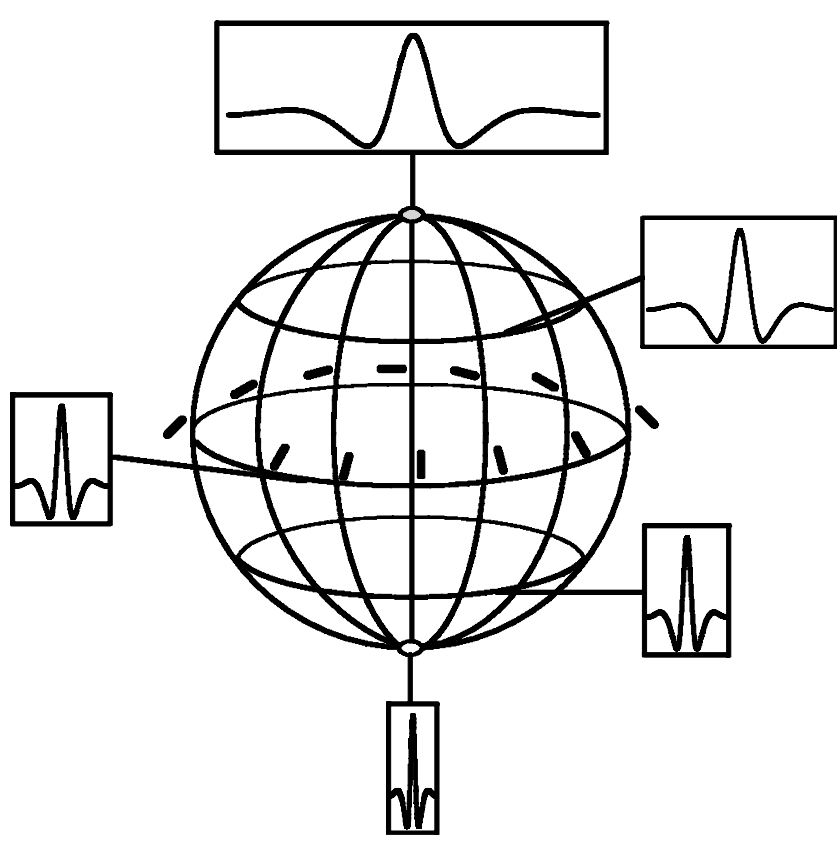

Figure 7. A spherical network topology. High and low spatial-frequency pinwheels are located at the poles of the sphere.

$a(\theta, \phi)=R_{0}+3 R_{1} \sum_{m=0, \pm} f_{m}(\Theta, \Phi) f_{m}(\theta, \phi)$.

Since $\Sigma_{m} f_{m}(\Theta, \Phi)^{2}=1$, we deduce that the gain is

$G=(C-\kappa)^{-1}\left[\frac{C(1-\varepsilon)-\kappa}{1-W_{0}}+\frac{C \varepsilon}{1-W_{1} / 3}\right]$.

In terms of the effective stimulus tuning

$\Gamma=\frac{\varepsilon C}{C-\kappa}$

we can re-express the gain as

$G=\frac{1-\Gamma}{1-W_{0}}+\frac{\Gamma}{1-W_{1} / 3}$.

Note that in the absence of any tuning or bias in the LGN input $(\varepsilon=0)$, we have $\Gamma=0$ and the broad activity profile reduces to the homogeneous state

$a(\theta, \phi)=\frac{C-\kappa}{1-W_{0}}$

with gain $G=1 /\left(1-W_{0}\right)$.

The existence and stability of a broad activity profile will depend on both $\Gamma$ and the weights $W_{0}, W_{1}$. First, since $a_{\mathrm{m} \text { in }}=R_{0}-3 R_{1}$ must be positive we require $\Gamma<\Gamma_{\mathrm{c}}$ where

$\frac{1}{\Gamma_{\mathrm{c}}}=1+\frac{1-W_{0}}{1-W_{1} / 3}$.

(When $\Gamma>\Gamma_{\mathrm{c}}$ the state is narrowly tuned, see below.) Second, a simple linear stability analysis shows that the broad activity profile is only asymptotically stable provided that

$W_{0}<1, W_{1}<3$.

At $W_{0}=1$ the system undergoes a bulk amplitude instability in which the activity across the network uniformly diverges. However, at $W_{1}=3$ there is a pattern-forming

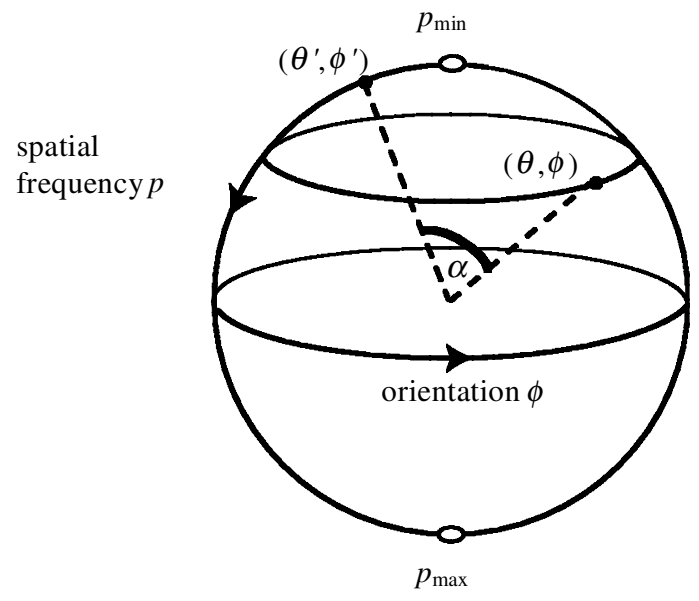

Figure 8. Spherical network topology. Orientation and spatial-frequency labels are denoted by $(\phi, p)$ with $0 \leqslant \phi<\pi$ and $p_{\min } \leqslant p \leqslant p_{\text {max }}$

instability associated with the bifurcation to a narrowly tuned or localized state. Indeed, as we establish below, when the spatial modulation of cortical recurrent interactions is sufficiently large, such a localized state can emerge spontaneously from the homogeneous state in the absence of any bias from the LGN input $(\varepsilon=0)$.

\section{(b) Narrow activity profile}

To simplify our analysis, we assume for the moment that the centre of the activity profile is fixed at the low frequency pinwheel, that is, $\Theta=0$. (The general solution can then be generated by carrying out an $S O(3)$ rotation on the sphere.) In this particular case, a state is narrowly tuned if there exists $\theta_{c}<\pi$ such that $a(\theta, \phi)=0$ for all $\theta_{\mathrm{c}} \leqslant \theta \leqslant \pi, 0 \leqslant \phi<\pi$. The cut-off angle $\theta_{\mathrm{c}}$ satisfies the equation

$I_{0}+\sum_{m} I_{1}^{m} f_{m}\left(\theta_{c}, \phi\right)=0,0 \leqslant \phi<\pi$.

Taking moments of the fixed point equation (3.9) with respect to the zeroth and first order spherical harmonics,

$$
\begin{aligned}
R_{0}= & I_{0} \int_{0}^{\theta_{c}} \int_{0}^{\pi} \mathscr{D}(\theta, \phi) \\
& +\sum_{m=0, \pm} I_{1}^{m} \int_{0}^{\theta_{c}} \int_{0}^{\pi} f_{m}(\theta, \phi) \mathscr{D}(\theta, \phi)
\end{aligned}
$$

and

$$
\begin{aligned}
R_{1}^{n}= & I_{0} \int_{0}^{\theta_{c}} \int_{0}^{\pi} f_{n}(\theta, \phi) \mathscr{D}(\theta, \phi) \\
& +\sum_{m=0, \pm} I_{1}^{m} \int_{0}^{\theta_{c}} \int_{0}^{\pi} f_{n}(\theta, \phi) f_{m}(\theta, \phi) \mathscr{D}(\theta, \phi)
\end{aligned}
$$

and performing the integration over $\theta, \phi$ then gives

$R_{0}=\frac{I_{0}\left[1-\cos \theta_{\mathrm{c}}\right]}{2}+\frac{I_{1}^{0}\left[1-\cos ^{2} \theta_{\mathrm{c}}\right]}{8}$

$R_{1}^{0}=\frac{I_{0}\left[1-\cos 2 \theta_{\mathrm{c}}\right]}{8}+\frac{I_{1}^{0}\left[1-\cos ^{3} \theta_{\mathrm{c}}\right]}{6}$

and 
$R_{1}^{ \pm}=\frac{I_{1}^{ \pm}\left[2-3 \cos \theta_{\mathrm{c}}+\cos ^{3} \theta_{\mathrm{c}}\right]}{12}$.

It is useful to introduce the functions

$$
\mathscr{A}_{0}\left(\theta_{\mathrm{c}}\right)=\frac{1-2 \cos \theta_{\mathrm{c}}+\cos ^{2} \theta_{\mathrm{c}}}{4}
$$

and

$$
\mathcal{L}_{1}\left(\theta_{\mathrm{c}}\right)=\frac{2-3 \cos \theta_{\mathrm{c}}+\cos ^{3} \theta_{\mathrm{c}}}{12} .
$$

Since $f \pm(0, \Phi)=0$ for all $\Phi$, it follows from equations (3.6) and (3.25) that

$R_{1}^{ \pm}\left[1-W_{1} \mathcal{A}_{1}\left(\theta_{\mathrm{c}}\right)\right]=0$.

Provided that $\mathrm{W}_{1} \mathscr{l}_{1}\left(\theta_{\mathrm{c}}\right) \neq 1$, we deduce that $R_{1}^{ \pm}=0$ and hence $I_{1}^{ \pm}=0$. Setting $I_{1}^{0}=I_{1}$ and $R_{1}^{0}=R_{1}$, the condition for $\theta_{c}$ reduces to

$I_{0}+I_{1} \cos \theta_{\mathrm{c}}=0$,

with (see equations (3.23) and (3.24))

$I_{0}=C(1-\varepsilon)+W_{0} R_{0}-\kappa, I_{1}=C \varepsilon+W_{1} R_{1}$.

Substituting into equations (3.23) and (3.24) gives $R_{0}=\mathcal{A}_{0}\left(\theta_{\mathrm{c}}\right) I_{1}$ and $R_{1}=\mathcal{A}_{1}\left(\theta_{\mathrm{c}}\right) I_{1}$ so that

$R_{0}=\frac{\varepsilon C \mathscr{C}_{0}\left(\theta_{\mathrm{c}}\right)}{1-W_{1} \mathscr{\iota}_{1}\left(\theta_{\mathrm{c}}\right)}$

and

$R_{1}=\frac{\varepsilon C \mathscr{A}_{1}\left(\theta_{\mathrm{c}}\right)}{1-W_{1} \mathscr{l}_{1}\left(\theta_{\mathrm{c}}\right)}$.

Given the critical angle $\theta_{c}$ and the effective input $I_{1}$, the resulting localized state takes the form

$a(\theta, \phi)=\left[I_{1}\left(\cos \theta-\cos \theta_{c}\right)\right]_{+}$

when centred about the $\Theta=0$ pinwheel. The corresponding gain defined by equation (3.10) is

$G=\frac{I_{1}\left(1-\cos \theta_{\mathrm{c}}\right)}{C-\kappa}$.

By performing an $S O(3)$ rotation, it immediately follows that a localized state centred at the point $(\Theta, \Phi)$ on the sphere is

$a(\theta, \phi)=\left[I_{1}\left(\sum_{m=0, \pm} f_{m}(\Theta, \Phi) f_{m}(\theta, \phi)-\cos \theta_{\mathrm{c}}\right)\right]_{+}$.

Thus, $a$ is only non-zero if the angular separation of $(\theta, \phi)$ from $(\Theta, \Phi)$ is less than the critical angle $\theta_{c}$. It follows that the boundary of the localized state $\theta=\theta_{0}(\phi)$ is given by the equation

$$
\sum_{m=0, \pm} f_{m}(\Theta, \Phi) f_{m}\left(\theta_{0}(\phi), \phi\right)=\cos \theta_{c} .
$$

We now determine properties of the localized state in different parameter regimes using a similar analysis to that of the ring model (Hansel \& Sompolinsky 1997).

(i) Weak cortical modulation $\left(W_{0}<1, W_{1}<3\right)$

For sufficiently weak cortical modulation, as defined by the condition $W_{1}<3$, a non-trivial activity profile only exists in the presence of a biased LGN input $(\varepsilon>0)$. Whether or not this state is broadly or narrowly tuned will depend on the stimulus parameter $\Gamma$. We have already established that the broadly tuned state exists only if $\Gamma<\Gamma_{\mathrm{c}}$ (see equation (3.18)). However, when $\Gamma>\Gamma_{\mathrm{c}}$ there exists a narrowly tuned state with critical angle $\theta_{\mathrm{c}}$ determined self-consistently from equations (3.29) and (3.30),

$$
\begin{aligned}
-\cos \theta_{\mathrm{c}} \equiv & \frac{I_{0}}{I_{1}}=\frac{C(1-\varepsilon)-\kappa}{C \varepsilon}\left[1-W_{1} \mathcal{A}_{1}\left(\theta_{\mathrm{c}}\right)\right] \\
& +W_{0} \mathscr{C}_{0}\left(\theta_{\mathrm{c}}\right),
\end{aligned}
$$

that can be rearranged to give

$\frac{1}{\Gamma}=1-\frac{W_{0} \mathscr{A}_{0}\left(\theta_{\mathrm{c}}\right)+\cos \theta_{\mathrm{c}}}{1-W_{1} \mathcal{A}_{1}\left(\theta_{\mathrm{c}}\right)}$.

Note that $\theta_{\mathrm{c}} \leqslant \pi$ for $\Gamma \geqslant \Gamma_{\mathrm{c}}$. In figure 10 we plot the critical angle $\theta_{\mathrm{c}}$ as a function of $\Gamma$. The corresponding gain of the localized state is

$G=\Gamma\left[\frac{1-\cos \theta_{c}}{1-W_{1} \mathcal{A}_{1}\left(\theta_{c}\right)}\right]$

where we have used equations (3.34) and (3.30).

It follows from equation (3.18) that if $W_{0}, W_{1} \approx 0$ then $\Gamma_{\mathrm{c}} \approx 0.5$ so that a stimulus with $\varepsilon<1 / 2$ and contrast $\mathrm{C} \gg \kappa$ will necessarily generate a broad activity profile. Introducing global inhibition by taking $W_{0}<0$ and $W_{1} \approx 0$ can sharpen the response by lowering $\Gamma_{\mathrm{c}}: \Gamma_{\mathrm{c}}$ $\approx 1 /\left(2+\left|W_{0}\right|\right)$. However, the gain is also lowered when the level of inhibition is increased since $G \approx \Gamma$ (1 $\left.-\cos \theta_{c}\right)$ and the cortical inhibition reduces $\theta_{c}$. Increasing the degree of cortical modulation $W_{1}$ for fixed $W_{0}$ also reduces $\Gamma_{\mathrm{c}}$ such that beyond the critical value $W_{1}=3$ we have $\Gamma_{c}=0$ and a localized state can be generated even in the absence of a feed-forward bias $\varepsilon$.

(ii) Marginal phase and strong cortical modulation ( $\varepsilon=0$, $W_{0}<W_{c}, W_{1}>3$ )

When $W_{1}>3$ the unique broadly tuned state (equation (3.13)) is unstable, so that any non-homogeneous state must be narrowly tuned. In the absence of an LGN bias $(\varepsilon=0)$ the former reduces to an unstable homogeneous state (equation (3.17)). Inspection of equation (3.32) shows that a localized state persists when $\varepsilon=0$ provided that

$1=W_{1} \mathscr{A}_{1}\left(\theta_{\mathrm{c}}\right)$.

Since $\mathscr{A}_{1}\left(\theta_{\mathrm{c}}\right)<1 / 3$ for $0 \leqslant \theta_{\mathrm{c}} \leqslant \pi$, it follows that $W_{1}>$ 3 is a necessary condition for a narrowly tuned activity profile to occur when $\varepsilon=0$. The location $(\Theta, \Phi)$ of the centre of the localized state is now arbitrary since the LGN input is homogeneous. In other words, there is a continuum of localized states on the sphere, which form a manifold of marginally stable fixed points, and the system is said to be in a marginal phase. In such a phase, a narrowly tuned state spontaneously breaks the underlying $S O$ (3) symmetry of the network, which is possible because the spatial modulation of the cortical interactions is sufficiently strong.

In the marginal phase, the critical angle $\theta_{c}$ is determined by equation (3.39) and is thus independent of $W_{0}$. Equations (3.31) and (3.32) imply that 
$\frac{R_{0}}{R_{1}}=\frac{\mathscr{A}_{0}\left(\theta_{\mathrm{c}}\right)}{\mathscr{A}_{1}\left(\theta_{\mathrm{c}}\right)}=W_{1} \mathscr{A}_{0}\left(\theta_{\mathrm{c}}\right)$.

Combining this with equations (3.30) and (3.20) and setting $\varepsilon=0$ then gives

$I_{1}=-\frac{C-\kappa}{\cos \theta_{\mathrm{c}}+W_{0} \mathscr{C}_{0}\left(\theta_{\mathrm{c}}\right)}$

and $R_{1}=I_{1} / W_{1}$. The corresponding gain (equation (3.34)) is

$G=-\frac{1-\cos \theta_{\mathrm{c}}}{\cos \theta_{\mathrm{c}}+W_{0} \mathscr{A}_{0}\left(\theta_{\mathrm{c}}\right)}$

that can be rewritten as

$G=-\frac{1-\cos \theta_{c}}{\ell_{0}\left(\theta_{c}\right)} \frac{1}{W_{c}-W_{0}}$

where

$W_{\mathrm{c}}=-\frac{\cos \theta_{\mathrm{c}}}{\Lambda_{0}\left(\theta_{\mathrm{c}}\right)}$.

Equation (3.43) implies that a second condition for the existence of a marginal localized state is that $W_{0}<W_{\text {c }}$. Performing a stability analysis shows that as $W_{0}$ approaches $W_{c}$ the system undergoes an amplitude instability analogous to that of the homogeneous state when $W_{0}=1$ and $W_{1}<3$ (see Appendix A). The phase diagram for the stability of the various states in the presence of a homogeneous input is shown in figure 11. The variation of the critical angle $\theta_{\mathrm{c}}$ and gain $G$ as a function of $W_{1}$ is plotted in figure 12 .

In the case of strong cortical modulation, the presence of a weak input bias $(0<\varepsilon \ll 1)$ will not affect the width of the activity profile but will explicitly break the hidden $S O$ (3) symmetry by locking the centre of the response $(\Theta, \Phi)$ to the peak of the LGN input. This establishes a recurrent mechanism for the joint contrast invariance of orientation and spatial-frequency tuning curves (see $\ 4$ ). Particular examples of localized states on the sphere are illustrated in figure 13 for $\theta_{c}=\pi / 3$ and various optimal spatial frequencies $\Theta$ and orientations $\Phi$. It can be seen that the differing solutions are related by a rotation of the sphere, which reflects the underlying $S O(3)$ symmetry. Finally, note that to simplify our analysis of the spherical model, we have considered a one-population model in which inhibitory and excitatory cell populations have been collapsed into a single equivalent population. Such a simplification greatly reduces the number of free parameters of the system. The basic insights gained from the onepopulation model can be used to develop the mean-field theory of a more realistic two-population model. This is presented in Appendix B.

\section{ORIENTATION AND SPATIAL-FREQUENCY TUNING CURVES}

Our mean-field analysis of the spherical model has generated exact solutions for two-dimensional localized states on the sphere, which correspond to population tuning surfaces for orientation and spatial-frequency preferences within a hypercolumn. A useful representation of the response is obtained by projecting the localized states onto the $(p, \phi)$-plane. Surface plots of the resulting activity profiles in the marginal phase are shown in figure 14 for $\Phi=90^{\circ}$ and either (a) $\Theta=\pi / 2$ (corresponding to an intermediate spatial frequency $p \approx 1.2$ cycles $\mathrm{deg}^{-1}$ ) or $(b)$ $\Theta=\pi / 3$ (corresponding to a lower spatial frequency $p \approx$ 1.2 cycles $\left.\mathrm{deg}^{-1}\right)$. Tuning curves for orientation and spatial frequency can then be extracted by taking vertical cross-sections through the tuning surface. Various examples are presented in figures 15-17. In particular, figure 15 illustrates the contrast invariance of the response with respect to both orientation and spatial frequency. In the marginal phase contrast, invariance is exact, since both the width $\theta_{\mathrm{c}}$ and the gain $G$ are independent of contrast (see equations (3.39) and (3.43)). Interestingly, approximate contrast invariance also holds for weak cortical modulation (small $W_{1}$ ), since $\theta_{\mathrm{c}}$ is a slowly varying function of the synaptic parameter $\Gamma$ over a broad parameter regime (see figure 11 ).

Figure 14 shows that projecting the spherical tuning surface onto the $(\theta, \phi)$-plane breaks the underlying $O(3)$ symmetry of the sphere. Consequently, the shape of the planar tuning surface varies under shifts in the location of the peak of the tuning surface. This distortion is a direct consequence of the existence of pinwheels, which are incorporated into our model using a spherical topology, and implies that the responses to orientation and spatial frequency are inseparable. That is, the activity profile cannot be written in the form $a(\theta, \phi)=U(\theta) V(\phi)$. However, we expect approximate separability to occur at intermediate spatial frequencies (away from the pinwheels). The non-separability of the response generates a behaviour that is consistent with some recent experimental observations.

(i) At high and low spatial frequencies (towards the pinwheels), there is a broadening of the tuned response to orientation. This is illustrated in figure $16 a$ where we plot orientation tuning curves $a(\Theta, \phi)$ as a function of $\phi$ for various optimal spatial frequencies $\Theta$. It can be seen that the width increases towards the low (and high) orientation pinwheel. No such broadening occurs for the corresponding spatial-frequency tuning curves as shown in figure $16 b$. In our model, the reduction of orientation selectivity around the pinwheels is an aggregate property of a population of cells. Interestingly, it has been found experimentally that individual neurons close to pinwheels are actually orientation selective (O'Keefe $e t$ al. 1998), but there is a broad distribution of orientation preferences within the pinwheel region so that the average response of the population is only weakly orientation selective. Note that our results differ from those of McLaughlin et al. (2000) who found a sharpening of orientation tuning near pinwheels. We attribute this difference to the $S O(3)$ symmetry we impose on the weighting function $w\left(\theta, \phi \mid \theta^{\prime}, \phi^{\prime}\right)$.

(ii) There is a systematic shift and narrowing of spatialfrequency tuning curves at non-optimal orientations - the shift is towards the closest pinwheel (see figure 17). There is some suggestion of spatial frequency shifts in recent optical imaging data (Issa et al. 2000). Note, however, that one difference between our model prediction and the data is that the latter appear to indicate a downward rather than 

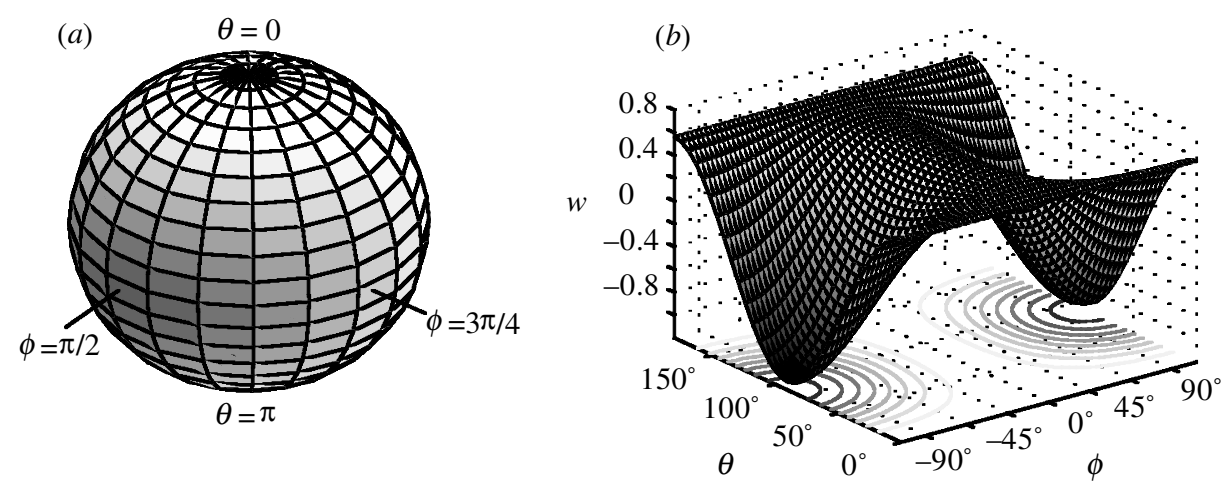

Figure 9. Two-dimensional plot of $w\left(\theta, \phi \mid \theta^{\prime}, \phi^{\prime}\right)$ given by $O(3)$ invariant weight distribution (equation $\left.(2.9)\right)$ with $W_{\mathrm{O}}=-1$, $W_{1}=1$ and $W_{n}=0$ for $n \geqslant 2$. We set $\phi^{\prime}=0, \theta^{\prime}=\theta$ and plot $w$ as a function of $\theta$ and $\phi$. (a) Contour plot of $w$ on the sphere with light and dark regions corresponding to excitation and inhibition, respectively. (b) Surface plot of $w$ in the $(\theta, \phi)$-plane.

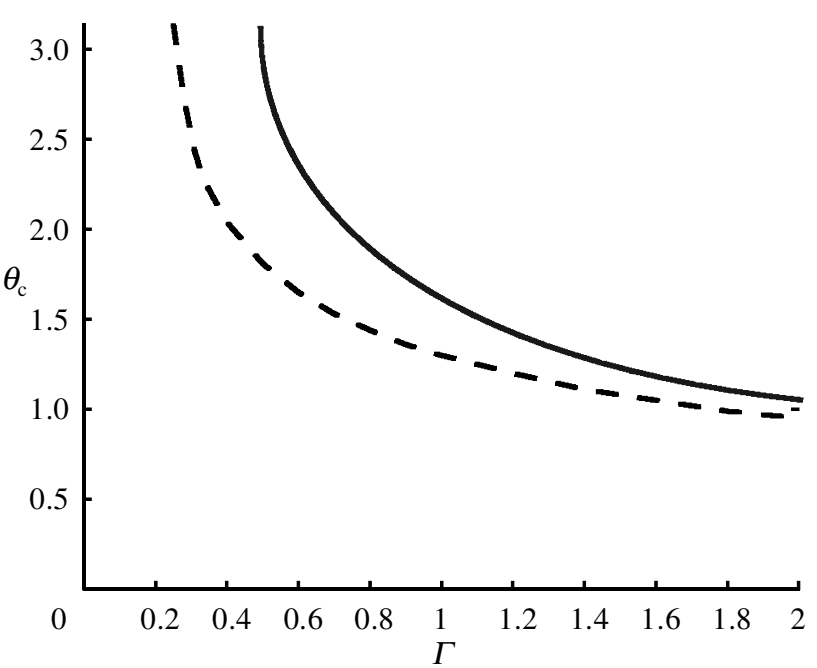

Figure 10. Critical angle $\theta_{c}$ for the width of the localized state as a function of the stimulus-tuning parameter $\Gamma$ in the case of weak cortical modulation $W_{1} \approx 0$. Solid line: $W_{\mathrm{O}}=0$; dashed line: $W_{\mathrm{O}}=-2$.

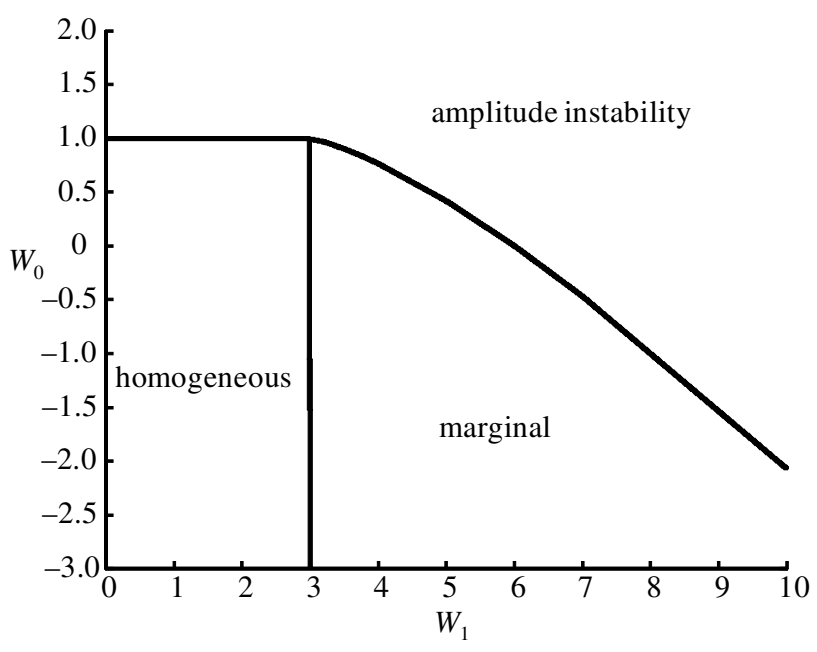

Figure 11. Phase diagram for the spherical model in the case of a homogeneous input $\varepsilon=0$.

an upward shift in response at high spatial frequencies. (A downward shift is also consistent with feed-forward receptive field properties, see figure

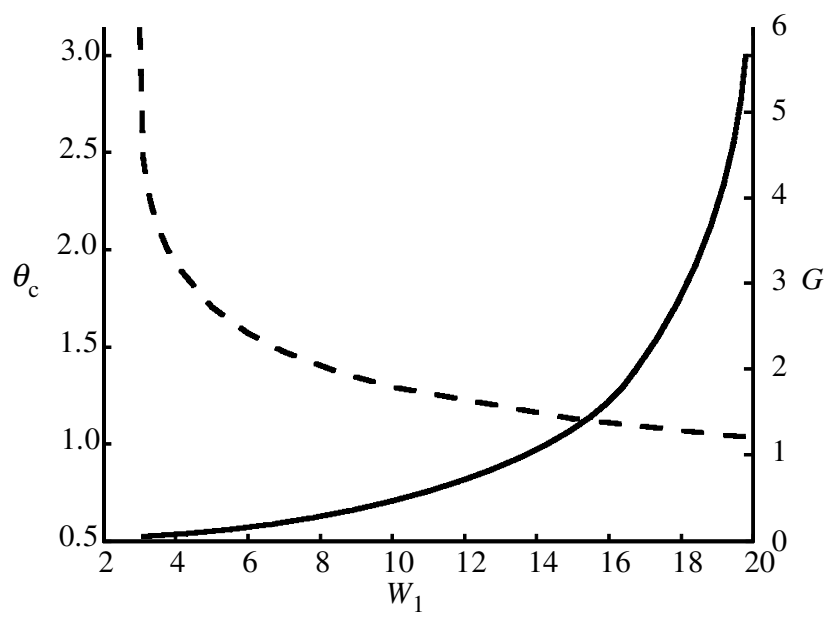

Figure 12. Variation of critical angle $\theta_{\mathrm{c}}$ (dashed line) and gain $G$ (solid line) as a function of cortical modulation $W_{1}$ in the case of homogeneous input $\varepsilon=0$. The gain is shown for $W_{\mathrm{O}}=-10$.

21.) We suggest in $₫ 7$ that the downward shift could be reversed by cortico-geniculate feedback (after some delay).

Another useful representation of the response is to consider contour plots of the activity profile in the $(\theta, \phi)$-plane as shown in figure 18 . Here, we use polar coordinates with radius $\theta$ and polar angle $\phi$. This figure further illustrates the non-separability of the response. We define $\Delta \theta$ as the width of the activity profile at the optimal orientation $\Phi$ and $\Delta \phi$ as the width of the activity profile at the optimal spatial frequency $\Theta$. It follows from equation (3.35) that $\Delta \theta=2 \theta_{c}$, irrespective of the position of the centre of the localized state. However, $\Delta \phi$ varies with the optimal frequency $\Theta$, reaching a minimum at $\Theta=\pi / 2$. Sufficiently close to the pinwheels, $\Theta<\theta_{\mathrm{c}} / 2$ or $\Theta>\pi-\theta_{\mathrm{c}} / 2$, we have $\Delta \phi=\pi$, which implies that although the response is localized on the sphere it is broadly tuned for orientation. Finally, in figure 19 we show a log-polar plot of various localized responses, which is at least suggestive of the single cell data reproduced in figure 3 . We select a narrow tuning width for ease of illustration since the data in figure 3 are thresholded. 

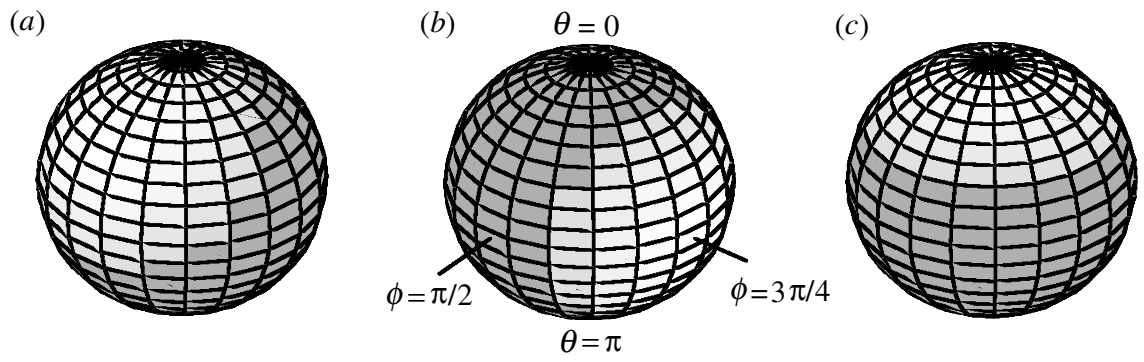

Figure 13. Two-dimensional plot of the tuning surface on the sphere associated with the localized solution (equation (3.35)). The activity $a(\theta, \phi)$ is plotted as a function of $(\theta, \phi)$ for fixed width $\theta_{\mathrm{c}}=\pi / 3$ and various optimal spatial frequencies $\Theta$ and orientations $\Phi:$ : (a) $\Theta=\pi / 4, \Phi=90^{\circ}$. (b) $\Theta=\pi / 2, \Phi=135^{\circ}$. (c) $\Theta=0, \Phi=0^{\circ}$. Light and dark regions denote high and low activities, respectively. The figures are related to each other by a rotation of the sphere.
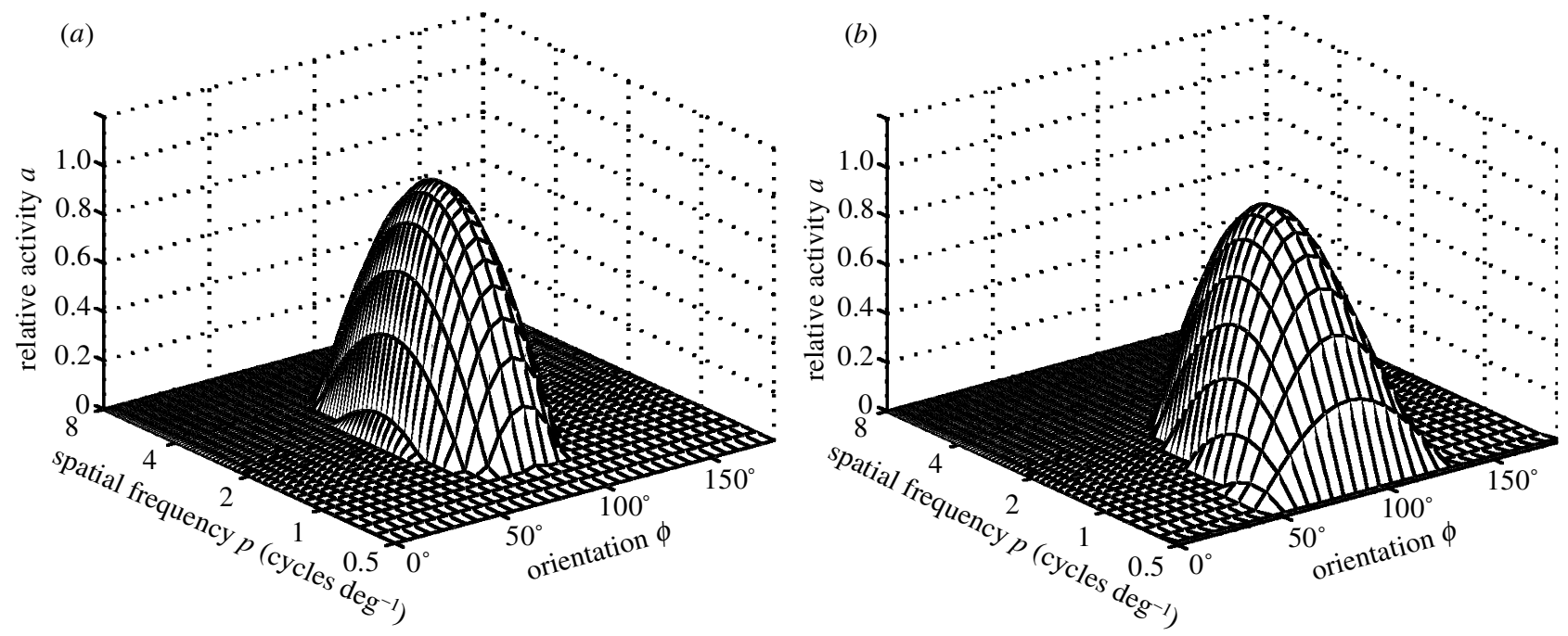

Figure 14. Plot of localized tuning surface in the $(p, \phi)$-plane in response to a weakly biased LGN input $(\varepsilon \ll 1)$ with $\Phi=90^{\circ}$ and $(a) \Theta=\pi / 2(b) \Theta=\pi / 3$. The width of the localized state is taken to be $\theta_{\mathrm{c}}=\pi / 3$. The activity $a$ is shown relative to its maximal value. We have assumed that $\theta$ is related to spatial frequency $p$ according the equation (2.1) with $p_{\min }=0.5$ cycles $\mathrm{deg}^{-1}$ and $p_{\max }=8$ cycles $\mathrm{deg}^{-1}$.
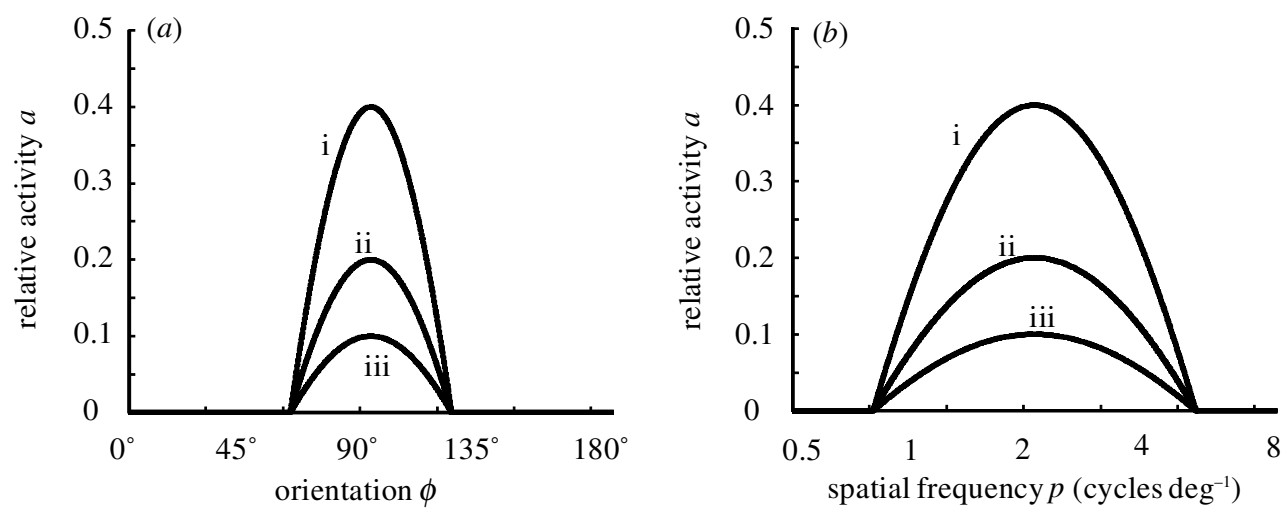

Figure 15. Contrast invariance of $(a)$ orientation and $(b)$ spatial-frequency tuning curves for $W_{1}=19.2$ and $W_{\mathrm{O}}=-10$ and a homogeneous input $(\varepsilon=0)$. The critical angle $\theta_{\mathrm{c}}=\pi / 3$ and the gain $G=4$. Curves correspond to contrasts (i) $C=0.2$, (ii) $C=0.1$, and (iii) $C=0.05$ relative to threshold $\kappa$.

We emphasize that the results presented in this section describe the response of a cortical hypercolumn to a fixed visual stimulus (population tuning curves) rather than the response of a single cell to a range of stimuli (single-cell tuning curves). The non-separability arising from the pin- wheels is thus a population effect and may be reduced or even absent at the single-cell response. Interestingly, recent single-cell recordings suggest that there is approximate separability of orientation and spatial-frequency tuning curves except at low and high spatial frequencies 
(a)

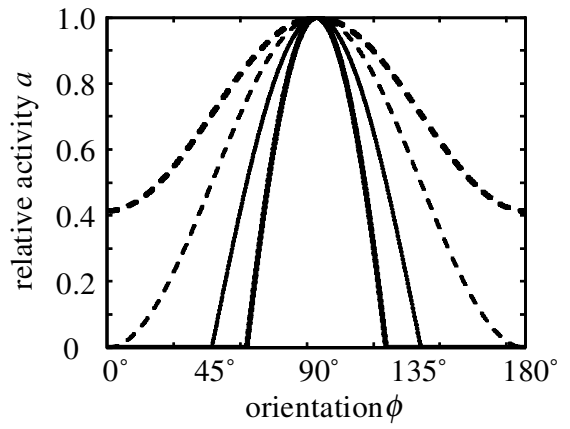

(b)

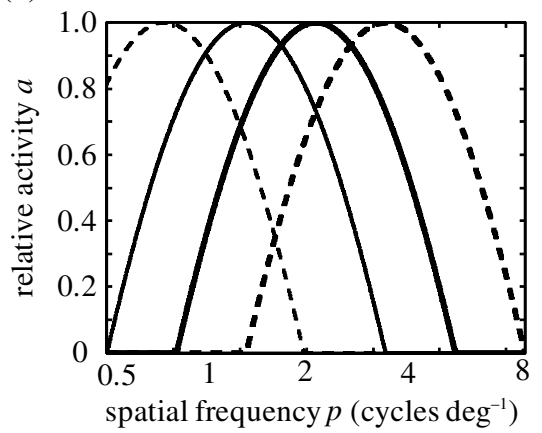

Figure 16. (a) Orientation tuning curves showing broadening as the optimal spatial frequency $\Theta$ changes from intermediate to high or low spatial frequencies: $\Theta=\pi / 6$ (thin dashed curve), $\Theta=\pi / 3$ (thin solid curve), $\Theta=\pi / 2$ (thick solid curve) and $\Theta=\pi / 8$ (thick dashed curve). The optimal orientation is fixed at $\Phi=90$ and $\theta_{c}=\pi / 3$. The activity $a$ is shown relative to its maximal value. (b) Spatial frequency tuning curves showing invariance of the degree of tuning with respect to $\Theta$. Same parameter values as (a) except $\Theta=2 \pi / 3$ (thick dashed curve). We have assumed that $\theta$ is related to $p$ according to equation (2.1) with $p_{\text {min }}=0.5$ cycles $\operatorname{deg}^{-1}$ and $p_{\max }=8$ cycles $\mathrm{deg}^{-1}$.
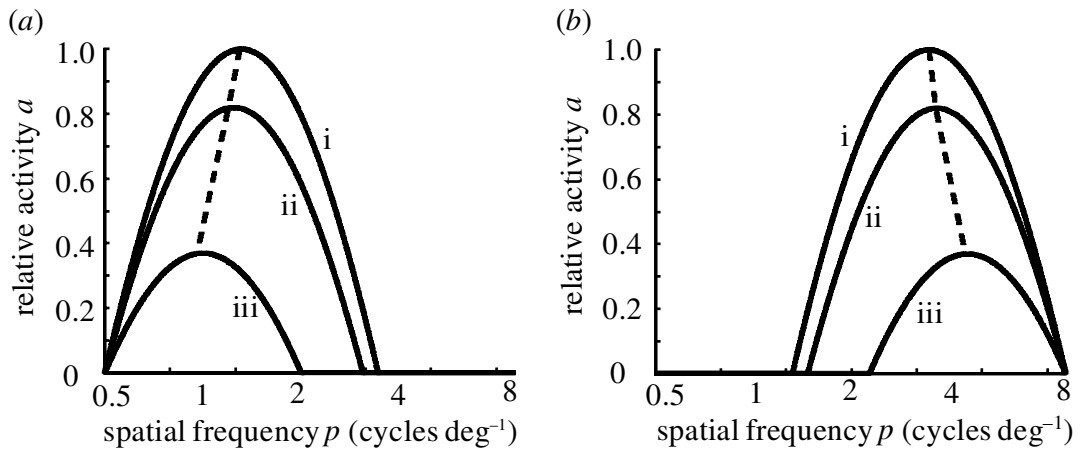

Figure 17. Spatial-frequency tuning curves $a(\theta, \phi)$ as a function of $\theta$ for various orientations $\phi=\Phi+\delta \phi$ : (i) $\delta \phi=0^{\circ}$, (ii) $\delta \phi=14^{\circ}$ (iii) $\delta \phi=28^{\circ}$. In the case of $(a)$ a low optimal frequency $\Theta=\pi / 3$ there is a downward shift in the peak of the response, whereas there is an upward shift in the case of $(b)$ a high optimal frequency $\Theta=2 \pi / 3$.

(Mazer et al. 2002), which is consistent with our population results.

\section{PART II: RECEPTIVE FIELDS AND CORTICO-GENICULATE FEEDBACK}

In Part II, we show that if the low-order spherical harmonic components of the filtered feed-forward input to a hypercolumn are amplified by recurrent interactions, then the spatial frequency at which the cortical response is optimal is shifted relative to the stimulus frequency-there is no corresponding shift in orientation. In other words, the network does not faithfully encode the stimulus spatial frequency. This shift in spatial frequency is not an artefact of the particular spherical network topology. A similar conclusion would obtain for any recurrent mechanism that amplifies both orientation and spatial-frequency components of the LGN input. We propose that the feedback pathway from V1 back to LGN, recently investigated in cats (Murphy et al. 1999), modulates LGN activity to produce a faithful encoding of spatial frequency. Using linear filter theory, we show that if the feedback from a cortical cell is taken to be approximately equal to the reciprocal of the corresponding feed-forward receptive field (in the two-dimensional Fourier domain), then the mismatch between the feed-forward and cortical frequency representations is eliminated (at least at the linear level). We pre- dict that for intermediate spatial frequencies, the corticogeniculate innervation pattern is oriented in a direction related to the orientation bias of its V1 origin. However, for high and low spatial frequencies, no direction of innervation should exist.

\section{FEED-FORWARD RECEPTIVE FIELDS}

One possible model of the two-dimensional receptive field of a simple cell (in retinal coordinates $\mathbf{r}=(x, y)$ ) is the difference of Gaussians (Hawken \& Parker 1987):

$$
\begin{aligned}
u(\mathbf{r})= & \frac{\sqrt{\kappa}}{2 \pi \sigma_{+}} \exp \left[-\frac{1}{2 \sigma_{+}^{2}}\left(\kappa^{2} x^{2}+y^{2}\right)\right] \\
& -\frac{\alpha}{2 \pi \sigma_{-}} \exp \left[-\frac{1}{2 \sigma_{-}^{2}}\left(x^{2}+y^{2}\right)\right] .
\end{aligned}
$$

This represents a centre-surround profile in which the excitatory centre is an ellipse with eccentricity $\kappa>1$ whose major axis runs along the $y$-direction. The inhibitory surround is taken to be circular but with a larger halfwidth, $\sigma_{-}>\sigma_{+}$. The parameter $\kappa$ is a measure of the degree of feed-forward orientation selectivity due to the alignment of LGN circular receptive fields along the vertical direction $(\phi=0)$. Taking the two-dimensional Fourier transform of $u$ gives 

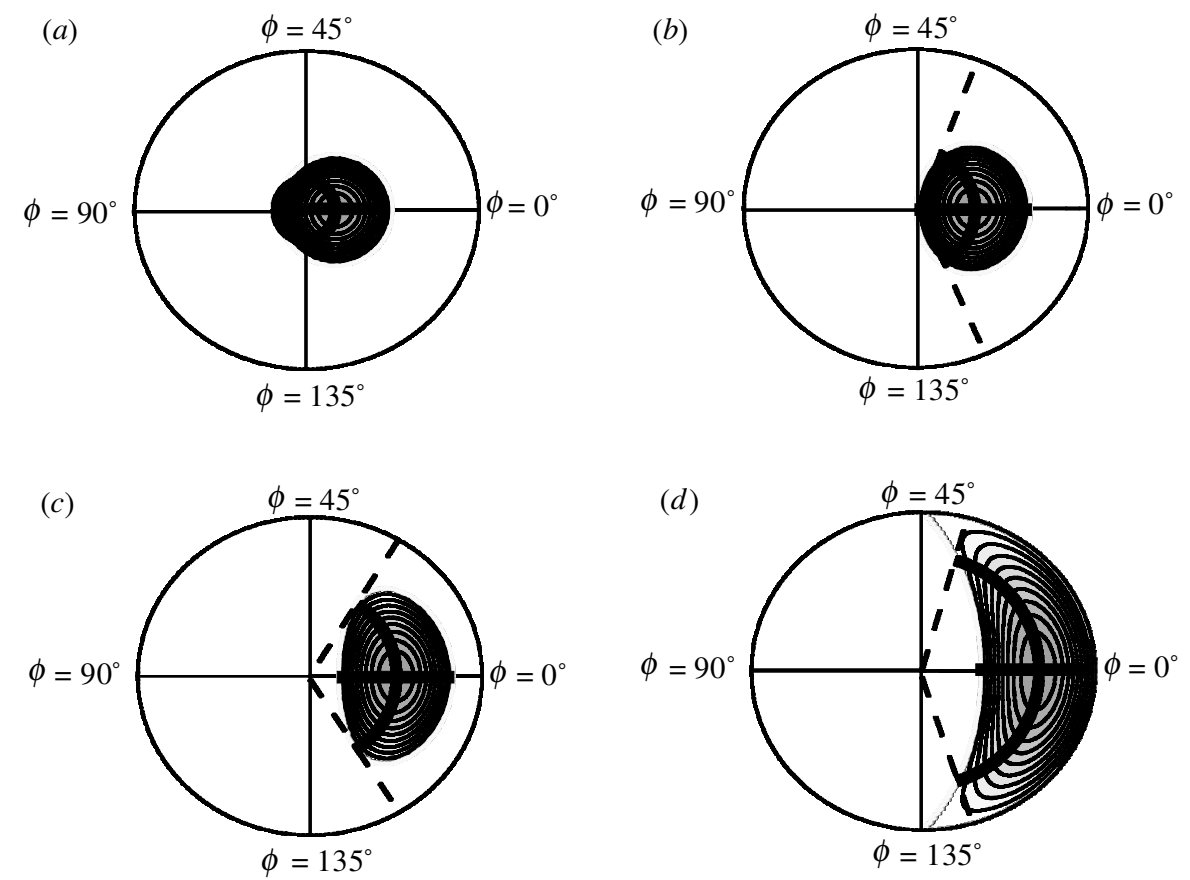

Figure 18. Polar plots of localized activity state $a(\theta, \phi)$ for fixed width $\theta_{\mathrm{c}}=\pi / 3$, fixed optimal orientation $\Phi=0^{\circ}$ and increasing optimal spatial frequency $\Theta$ : (a) $\Theta=\pi / 6,(b) \Theta=\pi / 3,(c) \Theta=\pi / 2$ and $(d) \Theta=2 \pi / 3$. Here, $\phi$ is taken to be the polar angle and $\theta$ the radius in the plane such that the origin represents the low-frequency pinwheel at $\theta=0$, whereas the outer circle represents the high-frequency pinwheel at $\theta=\pi$. Darker regions correspond to higher levels of activity. In each figure, $\Delta \theta=2 \theta_{\mathrm{c}}$ is indicated by the thick horizontal line and $\Delta \phi$ is indicated by the thick arc, reaching a minimum at $\Theta=\pi / 2$.

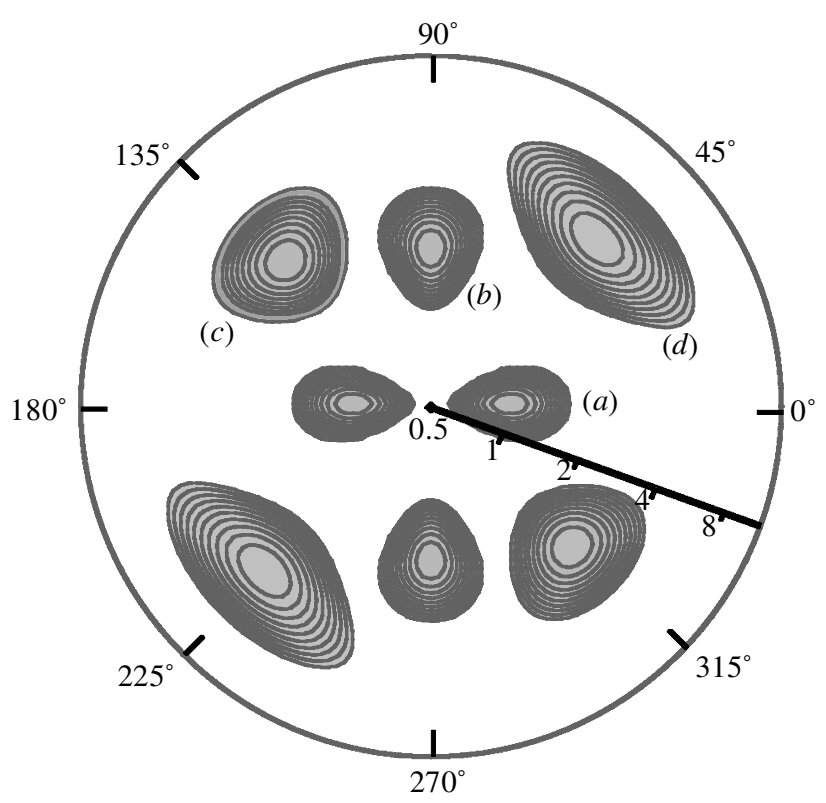

Figure 19. Log-polar plot of various localized activity states for fixed width $\theta_{\mathrm{c}}=\pi / 6$ and various optimal orientations $\Phi$ and spatial frequencies $P=\mathcal{L}^{-1}(\Theta)$ : (a) $P=1$ cycles $\mathrm{deg}^{-1}$, $\Phi=0^{\circ}$, (b) $P=2$ cycles $\operatorname{deg}^{-1}, \Phi=90^{\circ}$, (c) $P=3$ cycles $\operatorname{deg}^{-1}, \Phi=135^{\circ}$ and, (d) $P=4$ cycles $\operatorname{deg}^{-1}, \Phi=45^{\circ}$. Here $\phi$ is taken to be the polar angle and $\log _{2} p$ the radius.

$$
\begin{aligned}
U(\mathbf{k})= & \exp \left[-\frac{\sigma_{+}^{2} k^{2}}{2}\left(\kappa^{-2} \cos ^{2} \varphi+\sin ^{2} \varphi\right)\right] \\
& -\alpha \exp \left[-\frac{\sigma_{-}^{2} k^{2}}{2}\right],
\end{aligned}
$$

for $\mathbf{k}=(k, \varphi)$ in polar coordinates. The function $U$ has a maximum at $\mathbf{p}=(p, \phi)$ so that $U(\mathbf{p}) \geqslant U(\mathbf{k})$ for all $\mathbf{k}$, with $\phi=0, \pi$ and

$p=\sqrt{\frac{4}{\sigma_{-}^{2}-\kappa^{-2} \sigma_{+}^{2}} \ln \left[\frac{\sqrt{\alpha \kappa \sigma_{-}}}{\sigma_{+}}\right] .}$

Setting $\sigma_{+}=\sigma$ and $\sigma=\hat{\kappa} \sigma$ and taking $\hat{\kappa}, \kappa, \alpha$ to be fixed, it follows that the spatial-frequency preference $p$ is inversely proportional to the size $\sigma$ of the receptive field,

$p=\frac{A}{\sigma}, A=\sqrt{\frac{4}{\hat{\kappa}^{2}-\kappa^{-2}} \ln [\sqrt{\alpha \kappa \hat{\kappa}}]}$

and we can rewrite $u$ as

$$
\begin{aligned}
u(\mathbf{r} \mid p)= & \frac{p \sqrt{\kappa}}{2 \pi A} \exp \left[-\frac{p^{2}}{2 A^{2}}\left(\kappa^{2} x^{2}+y^{2}\right)\right] \\
& -\frac{\alpha p}{2 \pi \hat{\kappa} A} \exp \left[-\frac{p^{2}}{2 A^{2} \hat{\kappa}^{2}}\left(x^{2}+y^{2}\right)\right] .
\end{aligned}
$$

Now consider a cell with receptive field profile centred at the retinal coordinate $\hat{\mathbf{r}}$ with (feed-forward) orientation preference $\phi$ and spatial frequency preference $p$. Given a visual stimulus of intensity $i(\mathbf{r})$, the effective input from the LGN to the cell will be of the form

$h_{\mathrm{LGN}}(\hat{\mathbf{r}} \mid \mathbf{p})=\int i(\mathbf{r}) u(\hat{\mathbf{r}}-\mathbf{r} \mid p) d \mathbf{r}$

where $u(\mathbf{r} \mid \mathbf{p})=u\left(T_{\phi} \mathbf{r} \mid p\right)$ and

$T_{\phi}=\left(\begin{array}{cc}\cos \phi & -\sin \phi \\ \sin \phi & \cos \phi\end{array}\right)$. 

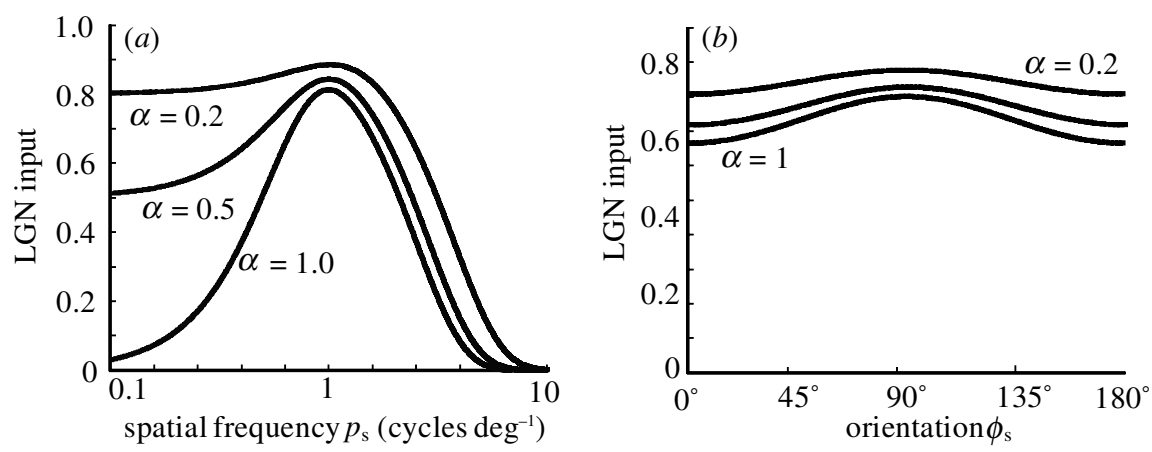

Figure 20. LGN input $h_{\mathrm{LGN}}$ to a single cell obtained by filtering a sinusoidal grating with the difference-of-Gaussians receptive field (5.5) for a range of stimulus spatial frequencies $p_{\mathrm{s}}$ and orientations $\phi_{\mathrm{s}}$ (with zero spatial phase). Parameters of the LGN receptive field are $\kappa=1.5, \hat{\kappa}=3$ with a variable level of surround inhibition $\alpha$. (a) Input (in units of the stimulus contrast $C_{\mathrm{s}}$ ) as a function of stimulus frequency $p_{\mathrm{s}}$ for a fixed spatial-frequency preference $p=1$ and $\phi=\phi_{\mathrm{s}}$. The units of spatial frequency are taken to be cycles $\mathrm{deg}^{-1}$. (b) The corresponding input as a function of stimulus orientation $\phi_{s}$ for a fixed orientation preference $\phi=90$ and $p=p_{\mathrm{s}}$.

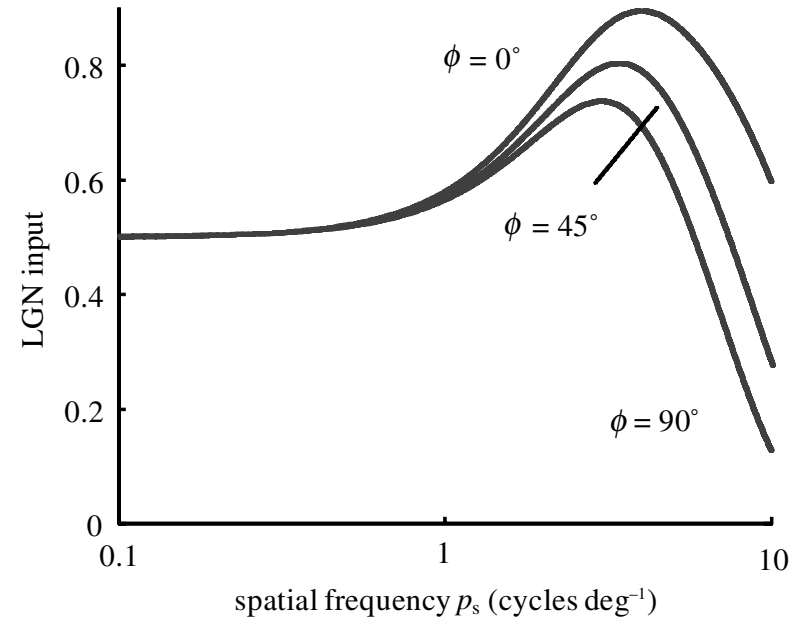

Figure 21. Shift in spatial-frequency peak of the LGN input at non-optimal orientation $\phi \neq \phi_{\mathrm{s}}$. Here, $p=4, \phi=0^{\circ}$ and $\kappa=2$. All other parameters as in figure 20 .

Taking the Fourier transform of equation (5.6) gives

$H_{\mathrm{LGN}}(\mathbf{k} \mid \mathbf{p})=I(\mathbf{k}) U(\mathbf{k} \mid \mathbf{p})$,

where

$$
\begin{aligned}
U(\mathbf{k} \mid \mathbf{p})= & \exp \left[-\frac{A^{2} k^{2}}{2 p^{2}}\left(\kappa^{-2} \cos ^{2}(\varphi-\phi)+\sin ^{2}(\varphi-\phi)\right)\right] \\
& -\alpha \exp \left[-\frac{\hat{\kappa}^{2} A^{2} k^{2}}{2 p^{2}}\right]
\end{aligned}
$$

In the particular case of a sinusoidal grating of contrast $C_{\mathrm{s}}$, spatial frequency $p_{\mathrm{s}}$ and orientation $\phi_{\mathrm{s}}$,

$i(\mathbf{r})=C_{\mathrm{s}} \cos \left[p_{\mathrm{s}}\left(x \cos \phi_{\mathrm{s}}+y \sin \phi_{\mathrm{s}}\right)\right]$,

we have

$h_{\mathrm{LGN}}(\hat{\mathbf{r}} \mid \mathbf{p})=C_{\mathrm{s}} U\left(\mathbf{p}_{\mathrm{s}} \mid \mathbf{p}\right) \cos \left(\mathbf{p}_{\mathrm{s}} \cdot \hat{\mathbf{r}}\right)$.

Thus, when the grating is centred in the receptive field of the neuron, so that $r=0, h_{\mathrm{LGN}}(0 \mid \mathbf{p})=C_{\mathrm{s}} U\left(\mathbf{p}_{\mathrm{s}} \mid \mathbf{p}\right)$, i.e. the resulting LGN input is given by the Fourier transform of the receptive field multiplied by the stimulus contrast $C_{\mathrm{s}}$, as expected.
In figure $20 a$ we plot the resulting LGN input as a function of stimulus frequency $p_{\mathrm{s}}$ for $\phi=\phi_{\mathrm{s}}, p=1$ and various levels of surround inhibition $\alpha$. It can be seen that for relatively low levels of inhibition, the LGN acts like a lowpass spatial-frequency filter with a shallow maximum at $p_{\mathrm{s}}=p$. When the inhibition is increased, however, the profile is sharpened and the LGN acts more like a band-pass filter. The corresponding input profile as a function of orientation preference $\phi$ is shown in figure $20 b$ for $\phi_{\mathrm{s}}=90^{\circ}$. The response has a shallow maximum at $\phi=\phi_{\mathrm{s}}$, with a relatively large constant background component that decreases with increasing surround inhibition and increasing $\kappa$. There is also a spatial frequency shift in the LGN input at non-optimal orientations $\left(\phi \neq \phi_{\mathrm{s}}\right)$, which is always to lower frequencies. This follows from equations (5.9) and (5.11), because when $\phi \neq \phi_{\mathrm{s}}$ there is an effective reduction in the anisotropy parameter $\kappa$ of the form $\kappa^{-2}>\kappa^{-2}\left(\cos ^{2}\left(\phi_{\mathrm{s}}-\phi\right)+\sin ^{2}\left(\phi_{\mathrm{s}}-\phi\right)\right)$. Such a reduction reduces the spatial frequency at which the input reaches a maximum, see equation (5.3), and this is true for all spatial frequencies as shown in figure 21 for $p=4$ cycles $\mathrm{deg}^{-1}$ This should be contrasted with the corresponding shift in the cortical response, which is to higher frequencies (see figure 17b).

\section{SPHERICAL HARMONIC PROJECTION OF THE LGN INPUT}

Now consider a cortical hypercolumn whose cells are parametrized by the orientation preference $\phi \in[0, \pi]$ and spatial-frequency preference $p \in\left[p_{\mathrm{m} \text { in }}, p_{\mathrm{max}}\right]$, with the pair $(p, \phi)$ determined by the feed-forward receptive field properties of the cells (see $₫ 5$ ). Following Part I, we assume that the network topology is a sphere with angular coordinates $(\theta, \phi)$, where $\theta$ is related to the spatialfrequency preference $p$ according to equation (2.1). We have already shown how amplification and rectification of certain spherical harmonic components of a weakly biased LGN input can generate orientation and spatial-frequency tuning. We are now interested in the consequences of selecting out these particular harmonic components without worrying about the additional rectification stage. Therefore, we restrict our analysis to linear theory and treat the cortex as a linear filter carrying out the transformation 
feed-forward receptive field V1

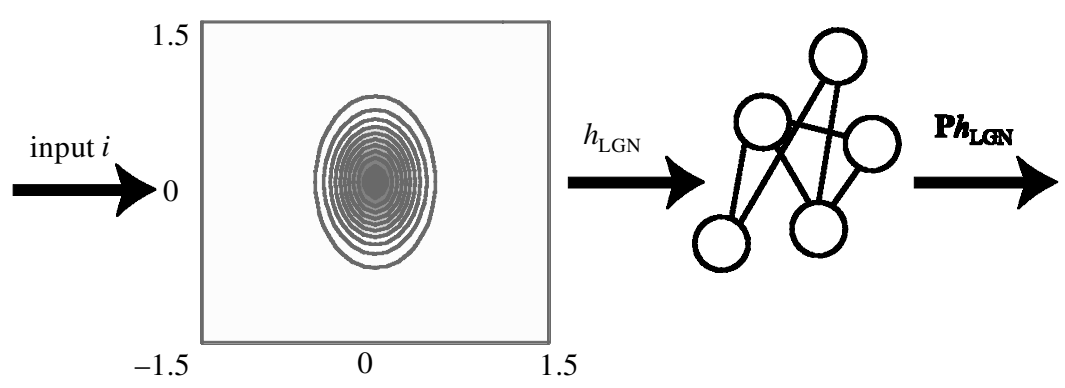

Figure 22. Schematic diagram of feed-forward pathways. A visual stimulus $i$ is convolved with a feed-forward receptive field $u$ to generate a cortical input $h_{\mathrm{LGN}}=u i$. (The convolution operator is defined in equation (7.3).) Recurrent interactions within V1 amplify low-order spherical harmonic components to generate response $h=\mathbf{P} h_{\mathrm{LGN}}$. The contour plot of a difference-ofGaussians receptive field profile is shown in retinal coordinates. The length scale is in units of the range of feed-forward excitation. Dark and light regions represent excitatory and inhibitory afferents, respectively. Parameters of the LGN receptive field are $\kappa=1.5, \hat{\kappa}=3$ and $\alpha=0.5$.

$h_{\mathrm{LGN}} \rightarrow \mathbf{P} h_{\mathrm{LGN}}$ where $\mathbf{P}$ denotes the projection onto the zeroth and first-order spherical harmonic components and $h_{\mathrm{LGN}}$ is the total feed-forward input from the LGN (see figure 22). (At first sight, this may be confusing since we took $h=\mathbf{P} h_{\mathrm{LGN}}$ to be the input to the cortex in Part I. We are essentially decomposing the operation of the cortex into two distinct parts: (i) selection through amplification $h_{\mathrm{LGN}} \rightarrow \mathbf{P} h_{\mathrm{LGN}}$ and (ii) tuning through amplification and rectification $\mathbf{P} h_{\mathrm{LGN}} \rightarrow$ a.)

Suppose, for the moment, that the receptive field centres of all neurons within a given hypercolumn are located at the same retinal coordinate $\hat{\mathbf{r}}$. Then $h_{\mathrm{LGN}}(\hat{\mathbf{r}} \mid \mathbf{p})$, for fixed $\hat{\mathbf{r}}$, determines the LGN input distribution across the hypercolumn. Projecting onto the first-order harmonics, it follows that

$$
\begin{gathered}
h(\theta, \phi) \equiv \mathbf{P} h_{\mathrm{LGN}}\left(\hat{\mathbf{r}} \mid \mathcal{L}^{-1}(\theta), \phi\right)=h_{0} \\
+\sum_{m=0, \pm} h_{1}^{m} f_{m}(\theta, \phi),
\end{gathered}
$$

where

$h_{0}=\frac{1}{2 \pi} \int_{0}^{\pi} \int_{0}^{\pi} h_{\mathrm{LGN}}\left(\hat{\mathbf{r}} \mid \mathcal{L}^{-1}(\theta), \phi\right) \sin \theta \mathrm{d} \theta \mathrm{d} \phi$

and

$h_{1}^{m}=\frac{3}{2 \pi} \int_{0}^{\pi} \int_{0}^{\pi} f_{m}(\theta, \phi) h_{\mathrm{LGN}}\left(\hat{\mathbf{r}} \mid \mathcal{L}^{-1}(\theta), \phi\right) \sin \theta \mathrm{d} \theta \mathrm{d} \phi$.

Note that for the resulting distribution $h(\theta, \phi)$ to be a well-defined function on the sphere, it must be independent of $\phi$ at $\theta=0, \pi$. Equations (5.6) and (5.9) then require that $\kappa=1$ at the pinwheels, in other words, the average orientation preference of receptive fields at the pinwheels must be zero. Hence, the existence of a nonzero preference away from the pinwheels implies that the orientation-selectivity parameter $\kappa$ has to be spatialfrequency dependent. For concreteness, we take

$\kappa=\kappa(\theta) \equiv \kappa_{0} \sin ^{2}(\theta)+\cos ^{2}(\theta)$,

with $\kappa_{0}>1$ so that the selectivity is maximal at intermediate spatial frequencies and zero at the pinwheels.

We now calculate $h(\theta, \phi)$ for a sinusoidal grating with stimulus frequency $p_{\mathrm{s}}$, orientation $\phi_{\mathrm{s}}$ and zero spatial phase $(\hat{\mathbf{r}}=0)$. We use the identities $\cos (2 \phi)=2 \cos ^{2} \phi-1$ $=1-2 \sin ^{2} \phi$ and

$\mathrm{e}^{x \cos 2 \phi}=I_{0}(x)+2 \sum_{n \geqslant 1} I_{n}(x) \cos (2 n \phi)$,

where $I_{n}(x)$ is the modified Bessel function of integer order $n$. Equation (5.9) can then be expanded as

$U(\mathbf{k} \mid \mathbf{p})=\sum_{n=0}^{\infty} U_{n}(k \mid p) \cos 2 n(\varphi-\phi)$,

where

$U_{0}(k \mid p)=\exp \left[-\frac{\boldsymbol{\kappa}^{+} A^{2} k^{2}}{2 p^{2}}\right] I_{0}\left(-\frac{\boldsymbol{\kappa}^{-} A^{2} k^{2}}{2 p^{2}}\right)-\alpha \exp \left[-\frac{\hat{\kappa}^{2} A^{2} k^{2}}{2 p^{2}}\right]$

and

$U_{n}(k \mid p)=2 \exp \left[-\frac{\boldsymbol{\kappa}^{+} A^{2} k^{2}}{2 p^{2}}\right] I_{n}\left(-\frac{\boldsymbol{\kappa}^{-} A^{2} k^{2}}{2 p^{2}}\right)$

for $n>0$ with $\kappa^{ \pm}=\left(1 \pm \kappa^{-2}\right) / 2$. Setting $h_{\mathrm{LGN}}(0 \mid \mathbf{p})$ $=C_{\mathrm{s}} U\left(\mathbf{p}_{\mathrm{s}} \mid \mathbf{p}\right)$ and using equations (6.1)-(6.3) and (6.6), we find that

$h_{0}=C_{\mathrm{s}} h_{0}\left(p_{\mathrm{s}}\right), h_{1}^{0}=C_{\mathrm{s}} h_{1}\left(p_{\mathrm{s}}\right)$

and

$h_{1}^{+}=C_{\mathrm{s}} h_{2}\left(p_{\mathrm{s}}\right) \cos \phi_{\mathrm{s}}, h_{1}^{-}=C_{\mathrm{s}} h_{2}\left(p_{\mathrm{s}}\right) \sin \phi_{\mathrm{s}}$,

with

$h_{0}\left(p_{\mathrm{s}}\right)=\frac{1}{2} \int_{p_{\text {min }}}^{p_{\text {max }}} U_{0}\left(p_{\mathrm{s}} \mid p\right) \sin (\mathcal{L}(p)) \mathrm{d} \mathcal{L}(p)$,

$h_{1}\left(p_{\mathrm{s}}\right)=\frac{3}{4} \int_{p_{\text {min }}}^{p_{\max }} U_{0}\left(p_{s} \mid p\right) \sin (2 \mathcal{L}(p)) \mathrm{d} \mathcal{L}(p)$

and

$h_{2}\left(p_{\mathrm{s}}\right)=\frac{3}{4} \int_{p_{\text {min }}}^{p_{\max }} U_{1}\left(p_{\mathrm{s}} \mid p\right) \sin ^{2}(\mathcal{L}(p)) \mathrm{d} \mathcal{L}(p)$. 


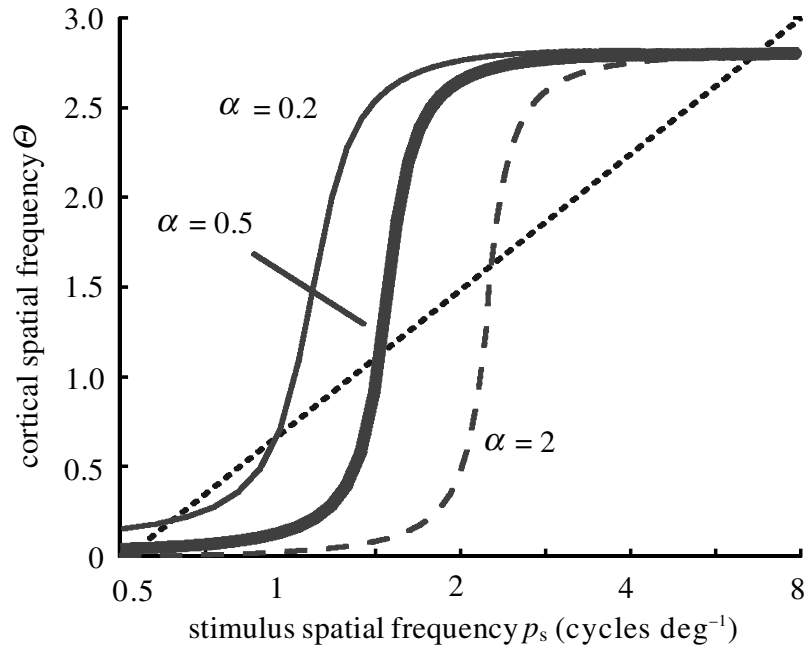

Figure 23. Plot of cortical spatial frequency $\Theta=\tilde{2}\left(p_{\mathrm{s}}\right)$ as a function of stimulus spatial frequency $p_{\mathrm{s}}$ for the differenceof-Gaussians receptive field with $\kappa_{0}=1.5, \hat{\kappa}=3$ and various levels of inhibition $\alpha$. The dotted line $\Theta=\tilde{2}\left(p_{\mathrm{s}}\right)$ corresponds to a faithful encoding of spatial frequency.

Substitution of equations (6.9) and (6.10) into equation (6.1) recovers the form assumed for $h$ in equation (2.10) of Part I, namely,

$$
\begin{aligned}
h(\theta, \phi)= & C\left[(1-\varepsilon)+\varepsilon \sum_{m=0, \pm} f_{m}(\Theta, \Phi) f_{m}(\theta, \phi)\right] \\
= & C(1-\varepsilon)+\varepsilon C(\cos \Theta \cos \theta+\sin \Theta \sin \theta \\
& \cos (2[\phi-\Phi])),
\end{aligned}
$$

where

$\Theta=\mathscr{2}\left(p_{\mathrm{s}}\right), \Phi=\phi_{\mathrm{s}}$

$C(1-\varepsilon)=C_{\mathrm{s}} h_{0}\left(p_{\mathrm{s}}\right), C \varepsilon=C_{\mathrm{s}} \Gamma\left(p_{\mathrm{s}}\right)$

and

$\mathscr{Z}\left(p_{\mathrm{s}}\right)=\tan ^{-1} \frac{h_{2}\left(p_{\mathrm{s}}\right)}{h_{1}\left(p_{\mathrm{s}}\right)}, \Gamma\left(p_{\mathrm{s}}\right)=\sqrt{h_{1}\left(p_{\mathrm{s}}\right)^{2}+h_{2}\left(p_{\mathrm{s}}\right)^{2}}$.

The phase $\tilde{\mathscr{L}}\left(p_{\mathrm{s}}\right)$ is plotted as a function of stimulus frequency in figure 23 for various levels of feed-forward inhibition $\alpha$. This clearly shows that $\Theta=\tilde{2}\left(p_{\mathrm{s}}\right) \neq \mathscr{2}\left(p_{\mathrm{s}}\right)$-there is a strong magnification of the representation of spatial frequency in the intermediate range, with small changes in $p_{\mathrm{s}}$ inducing large changes in the location $\Theta$ of the peak of the tuned response. Thus, there is a mismatch between the spatial frequency encoded by the hypercolumn (given by $\Theta$ ) and the input spatial frequency $p_{\mathrm{s}}$ of the stimulus. In figure 24 we plot the variation of $h_{0}\left(p_{\mathrm{s}}\right)$ and $\Gamma\left(p_{\mathrm{s}}\right)$ with stimulus frequency. These functions determine the effective contrast $C$ and bias $\varepsilon$ according to equation (6.16) so that, in particular, the contrast $C=h_{0}\left(p_{\mathrm{s}}\right)+\Gamma\left(p_{\mathrm{s}}\right)$. In the mean-field analysis of $₫ 3$, we showed that under amplification and rectification a localized activity state is generated whose amplitude varies as $\varepsilon C$ (weak cortical modulation) or as $C$ (strong cortical modulation, weak bias 1). We see from figure 24 that the projection onto spherical harmonics leads to a non-trivial dependence of the response amplitude on stimulus frequency. This appears to be inconsistent with physiological (Issa et al. 2000) and psychophysical (De Valois \& De Valois 1988) data that indicate that the response amplitude is a unimodal function that peaks at a single intermediate frequency. Another interesting observation regarding figure 24 , is that the LGN bias $\varepsilon$ cannot be assumed small across the entire spatial-frequency range.

The origin of the mismatch $\Theta \neq 2\left(p_{\mathrm{s}}\right)$ is the assumption that recurrent cortical interactions amplify both orientation and spatial-frequency components of the LGN input. Such a mismatch would not occur if Fourier modes with respect to the orientation label $\phi$ alone were amplified, as in the ring model of orientation tuning (Ben-Yishai et al. 1995). In such a case, one can represent the effective LGN input for a fixed spatial frequency preference $p$ by

$h(\phi)=C(1-\varepsilon)+C \cos \left(2\left[\phi-\phi_{\mathrm{s}}\right)\right]$

with, see equation (6.6),

$C(1-\varepsilon)=U_{0}\left(p_{\mathrm{s}} \mid p\right), C \varepsilon=U_{1}\left(p_{\mathrm{s}} \mid p\right)$.

Suppose that the stimulus frequency $p_{\mathrm{s}}$ is fixed and we plot $U_{0,1}\left(p_{\mathrm{s}} \mid p\right)$ as a function of the spatial-frequency preference $p$. The results are shown in figure 25 . At the optimal orientation $\phi=\phi_{\mathrm{s}}$ the spatial-frequency dependence of the input is given by the effective contrast $C=U_{0}\left(p_{\mathrm{s}} \mid p\right)+U_{1}\left(p_{\mathrm{s}} \mid p\right)$. It can be seen from figure 25 that $C$ peaks when the spatial-frequency preference is approximately equal to the stimulus frequency, $p=p_{\mathrm{s}}$, so that the network response now faithfully encodes the stimulus. However, the resulting spatial-frequency tuning curves are neither sharply tuned nor contrast invariant. (These tuning curves are directly given by $C$ since there is no amplification with respect to $p$ ). One way to achieve more realistic tuning curves, is to posit that recurrent interactions also amplify spatial-frequency components of the LGN input along the lines of the spherical model. One then has to tackle the resulting mismatch between stimulus frequency and response frequency.

\section{RENORMALIZING THE LGN INPUT}

It follows from the above analysis that if the cortex amplifies the first-order spherical harmonic components of a stimulus, then to generate a faithful representation of spatial frequency, $\Theta=\mathscr{L}\left(p_{\mathrm{s}}\right)$, the LGN input cannot be determined only by the feed-forward receptive field properties of single neurons. In other words, another filtering operation, $\mathscr{Q}$, must exist that converts $\tilde{Z}\left(p_{\mathrm{s}}\right)$ into $\mathcal{L}\left(p_{\mathrm{s}}\right)$. Of course, an alternative possibility is that the proposed amplification mechanism is itself invalid. However, we expect a similar conclusion to hold for any feed-forward or recurrent mechanism that amplifies two-dimensional Fourier components of the stimulus-the basic problem lies with the fact that the response is inseparable with respect to the orientation and spatial frequency labels. It therefore remains to be discussed, what are the possible mechanisms for the filtering action $\mathscr{Q}$ that effectively renormalizes the feed-forward LGN input.

\section{(a) Feed-forward mechanisms}

One possible feed-forward mechanism is patch averaging. For simplicity, we have assumed that every cortical cell within a local patch has the same receptive field profile $u$, equation (5.5), with identical parameters $\alpha, \kappa, \hat{\kappa}$ and 

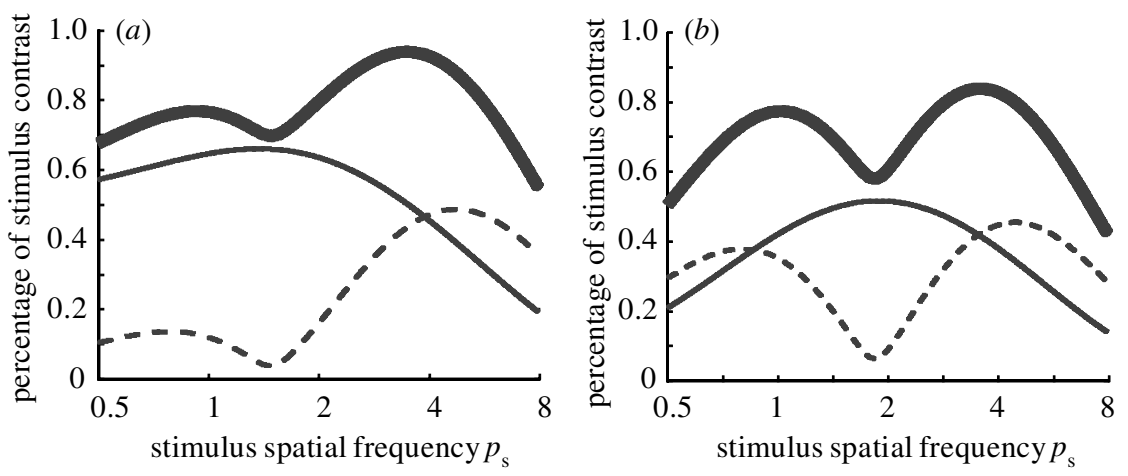

Figure 24. Plot of $h_{\mathrm{o}}\left(p_{\mathrm{s}}\right)$ (thin curve), $\Gamma\left(p_{\mathrm{s}}\right)$ (dashed curve) and the contrast $C$ (thick curve) as a function of stimulus spatial frequency $p_{\mathrm{s}}$ for $(a) \alpha=0.5(b) \alpha=1.0$. Other parameter values as in figure 23 .

(a)

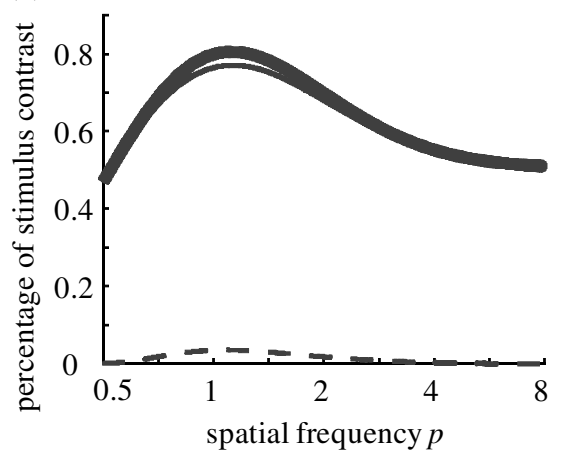

(b)

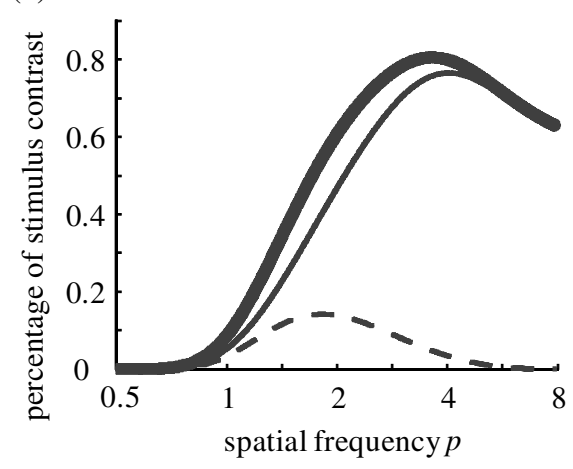

Figure 25. Plot of $U_{\mathrm{o}}\left(p_{\mathrm{s}} \mid p\right)$ (dashed curve), $U_{1}\left(p_{\mathrm{s}} \mid p\right)$ (thin curve) and contrast $C$ (thick curve) as a function of spatialfrequency preference $p$ for fixed stimulus frequency $p_{\mathrm{s}}$ : (a) $p_{\mathrm{s}}=1$ cycles $\mathrm{deg}^{-1}$ and $(b) p_{\mathrm{s}}=4.0 \mathrm{cycles}^{\mathrm{deg}}{ }^{-1}$. Other parameter values as in figure 23 for $\alpha=0.5$.

receptive field centres $\hat{\mathbf{r}}$. In reality, there will be a distribution of receptive fields so that the filter action $\mathscr{Q}$ could arise from some form of patch averaging. For example, figure 23 indicates that if there were some variation in the level of feed-forward inhibition $\alpha$, then this would smooth out the response. A more realistic source of variation is that of receptive field positions within each cortical column. Let the distribution of centres within a patch be $\rho(\hat{\mathbf{r}} \mid p)$, where the degree of scatter may depend on $p$, the spatial frequency preference of the patch. Equation (6.3) is then modified according to

$h_{1}^{m}=\frac{3}{2 \pi} \int_{0}^{\pi} \int_{0}^{\pi} f_{m}(\theta, \phi)\left[\int \mathrm{d} \hat{\mathbf{r}} \rho\left(\hat{\mathbf{r}} \mid \mathcal{L}^{-1}(\theta)\right) h_{\mathrm{LGN}}\left(\hat{\mathbf{r}} \mid \mathcal{L}^{-1}(\theta), \phi\right)\right]$

and similarly for equation (6.2). Such averaging may be expected to smooth cortical responses.

A second feed-foward mechanism is a non-trivial mapping of the cortical labels. The projection of the LGN input onto the low-order spherical harmonics given by equations (6.2) and (6.3) assumes that the cortical labels for orientation and spatial frequency $(p, \phi)$ are determined completely by properties of the feed-forward receptive fields (see $\ 5$ ). This is the classical Hubel-Wiesel mechanism for generating the feature preferences of a cell. We have shown that such identification leads to a mismatch in the representation of spatial frequency within the cortex. One possible way to eliminate such a mismatch is to allow for a non-trivial mapping between properties of the receptive field and the cortical labels that regularizes the projection of the LGN input and, hence, generates a faithful representation of spatial frequency. This mapping reflects the fact that the actual spatial frequency and orientation preference of a cell is determined by a combination of feed-forward and recurrent interactions. A renormalization scheme of this form would require the development of a pattern of innervation from LGN to cortex that involves some form of feedback from cortex to LGN to implement an error correcting procedure. But such feedback can itself provide a direct mechanism for renormalizing the LGN input, as we describe below.

\section{(b) Cortico-geniculate feedback}

We constructed a recurrent filter that converts the feedforward or bare receptive field $u$ into an effective or renormalized one, namely $u^{*}$, such that the renormalized LGN input

$h_{\mathrm{LGN}}^{*}(\hat{\mathbf{r}} \mid \mathbf{p})=\int i(\mathbf{r}) u^{*}(\hat{\mathbf{r}}-\mathbf{r} \mid \mathbf{p}) \mathrm{d} \mathbf{r}$

projects faithfully onto its spherical harmonic components. Inverse Fourier transforming the recurrent filter then determines the pattern of feedback connections from V1 to LGN. First, suppose that a cortical cell with receptive field centre $\hat{\mathbf{r}}$, spatial-frequency preference $p$ and orientation preference $\phi$, has a distribution of feedback connections $v(\mathbf{r}-\hat{\mathbf{r}} \mid \mathbf{p})$ to LGN cells that innervate cortical cells with the same feature preference and shifted 


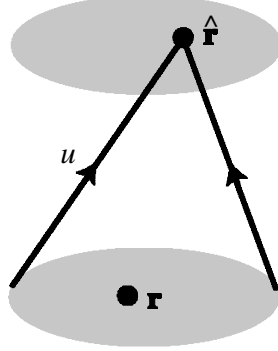

(a)

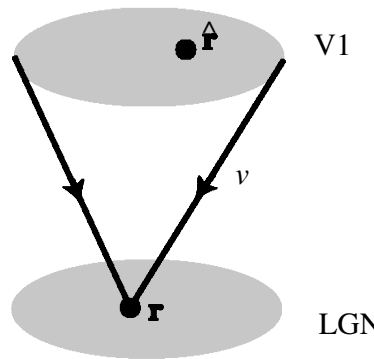

(b)
Figure 26. Schematic diagram showing reciprocally related regions in V1 and LGN. (a) Feed-forward projections and (b) feedback projections.

centre at $\mathbf{r}$, i.e. the LGN cells make cortical connections with a weighting function $u(\hat{\mathbf{r}}-\mathbf{r} \mid \mathbf{p})$. In other words, we assume that localized patches in the cortex and LGN are reciprocally related (Murphy et al., 1999; Guillery et al. 2001) (see figure 26). Such a principle also seems to hold with respect to feedback from extra-striate to striate areas (Angelucci et al. 2001).

Within the framework of linear filter theory, we assume the output activity of the cortex consists of the spherical harmonic components of the renormalized LGN input. (A more complete calculation would need to take into account amplification and rectification of $\mathbf{P} h_{\mathrm{LGN}}$.) We write this output activity in the form $\mathbf{P} u^{*} i(\hat{\mathbf{r}})$, where $i$ is the input stimulus and $f \circ g$ for arbitrary functions $f, g$ denotes the convolution

$[f \circ g](\mathbf{r})=\int_{\mathbf{R}^{2}} f(\mathbf{r}) g\left(\mathbf{r}-\mathbf{r}^{\prime}\right) \mathrm{d} \mathbf{r}^{\prime}$.

We then assume that (see figure 27)

$u^{*} i=u\left[i+v \circ \mathbf{P}\left[u^{*} \circ i\right]\right]$.

Taking the Fourier transform of this equation using the convolution theorem,

$U^{*}(\mathbf{k} \mid \mathbf{p}) I(\mathbf{k})=U(\mathbf{k} \mid \mathbf{p}) I(\mathbf{k})\left[1+V(\mathbf{k} \mid \mathbf{p}) \mathbf{P} U^{*}(\mathbf{k} \mid \mathbf{p})\right]$

for $\mathbf{k}=(k, \varphi)$ in polar coordinates. Rearranging this equation leads to the result

$V(\mathbf{k} \mid \mathbf{p})=\frac{U^{*}(\mathbf{k} \mid \mathbf{p})-U(\mathbf{k} \mid \mathbf{p})}{U(\mathbf{k} \mid \mathbf{p}) \mathbf{P} U^{*}(\mathbf{k} \mid \mathbf{p})}$.

As a further simplification, suppose $U^{*}-U=\mathbf{P}\left[U^{*}-U\right]$ so that

$V(\mathbf{k} \mid \mathbf{p})=\frac{1}{U(\mathbf{k} \mid \mathbf{p})}\left[1-\frac{\mathbf{P} U(\mathbf{k} \mid \mathbf{p})}{\mathbf{P} U^{*}(\mathbf{k} \mid \mathbf{p})}\right]$.

Both $\mathbf{P} U(\mathbf{k} \mid \mathrm{p})$ and $\mathbf{P} U^{*}(\mathbf{k} \mid \mathbf{p})$ can be expressed in terms of zeroth and first-order spherical harmonics:

$\frac{\mathbf{P} U(\mathbf{k} \mid \mathbf{p})}{\mathbf{P} U^{*}(\mathbf{k} \mid \mathbf{p})}=\left[\frac{C(k)}{C^{*}(k)}\right]$

$\times \frac{(1-\varepsilon(k))+\varepsilon(k)(\cos \Theta(k) \cos \theta+\sin \Theta(k) \sin \theta \cos (2[\phi-\varphi]))}{\left(1-\varepsilon^{*}(k)\right)+\varepsilon^{*}(k)\left(\cos \Theta^{*}(k) \cos \theta+\sin \Theta^{*}(k) \sin \theta \cos (2[\phi-\varphi])\right)}$.

The non-renormalized functions $C(k), \varepsilon(k), \Theta(k)$ satisfy equations (6.15) and (6.16). In particular, $\Theta(k)=\tilde{\mathcal{L}}(k)$. Hence, the feedback distribution $V$ is determined once we have specified the $k$-dependence of the renormalized functions $C^{*}(k), \varepsilon^{*}(k)$ and $\Theta^{*}(k)$. It also follows that the mis- match in spatial frequencies highlighted in $\$ 6$ is eliminated provided that $\Theta^{*}(k)=2(k)$.

Further insight into the nature of the feedback connections can be obtained under the additional assumption that $C \ll C^{*}$ over the frequency bandwidth of the hypercolumn. The lowest-order approximation is then

$V(\mathbf{k} \mid \mathbf{p}) \approx \frac{\mathscr{A}}{U(\mathbf{k} \mid \mathbf{p})}$,

where $\mathscr{A}<1$ is a constant. Using equation (6.6) and keeping only the lowest-order terms,

$$
\begin{aligned}
V(\mathbf{k} \mid \mathbf{p}) & \approx \frac{\mathscr{l}}{U_{0}(k \mid p)+U_{1}(k \mid p) \cos (2[\varphi-\phi])} \\
& \approx \frac{\ell}{U_{0}(k \mid p)}\left[1-\frac{U_{1}(k \mid p)}{U_{0}(k \mid p)} \cos (2[\varphi-\phi])\right] .
\end{aligned}
$$

Taking the inverse Fourier transform of this equation shows that

$v(\mathbf{r} \mid \mathbf{p})=\operatorname{Re} \int_{P_{\text {min }}}^{P_{\max }} \int_{0}^{2 \pi} V(\mathbf{k} \mid \mathbf{p}) \mathrm{e}^{i \mathbf{r} \cdot \mathbf{k}} \frac{k \mathrm{~d} k \mathrm{~d} \varphi}{4 \pi^{2}}$

where we have assumed that the feedback is restricted to lie within the frequency bandwidth $\left[p_{\mathrm{min}}, p_{\mathrm{max}}\right]$ of the hypercolumn. To evaluate this integral, introduce polar coordinates $x=r \cos \psi, y=r \sin \psi \quad$ so that $\mathbf{k} \cdot \mathbf{r}$ $=k r \cos (\varphi-\psi)$. The Bessel function expansion

$\cos (x \cos \psi)=\mathfrak{F}_{0}(x)+2 \sum_{n=1}^{\infty}(-1)^{n} \mathfrak{F}_{2 n}(x) \cos (2 n \psi)$

then gives

$v(\mathbf{r} \mid \mathbf{p})=v_{0}(r \mid p)+v_{1}(r \mid p) \cos (2[\phi-\psi])$

where

$v_{0}(r \mid p)=\int_{p_{\min }}^{p_{\max }} \frac{\mathcal{A}}{U_{0}(k \mid p)} \mathcal{F}_{0}(k r) \frac{k \mathrm{~d} k}{2 \pi}$,

$v_{1}(r \mid p)=\mathcal{l} \int_{p_{\min }}^{p_{\max }} \frac{U_{1}(k \mid p)}{U_{0}(k \mid p)^{2}} \mathcal{F}_{2}(k r) \frac{k \mathrm{~d} k}{2 \pi}$.

We have thus specified an approximate form of the recurrent filter that effectively renormalizes the LGN input. We see from equations (7.9) and (7.11) that this filter is approximately the inverse Fourier transform of the reciprocal of $U(\mathbf{k} \mid \mathbf{p})$, the Fourier transform of the feedforward receptive field $u(\mathbf{r} \mid p)$ located at $\mathbf{r}$ with spatial frequency bias $\mathbf{p}=(\phi, p)$. Figure 27 shows the form of such a filter in case the feed-forward filter is tuned to $\phi=0$. It will be seen that the patterned feedback found in the model appears to be consistent with that observed by Murphy et al. (1999) in that it depends on the orientation preference of its V1 origin.

\section{CROSS-ORIENTATION SUPPRESSION}

A consistent experimental finding is that when a hypercolumn is stimulated with a pair of orthogonal gratings or bars there is considerable suppression of the response to either stimulus. In particular, DeAngelis et al. (1992) show that this cross-orientation suppression originates within the receptive field of most cat neurons examined, 


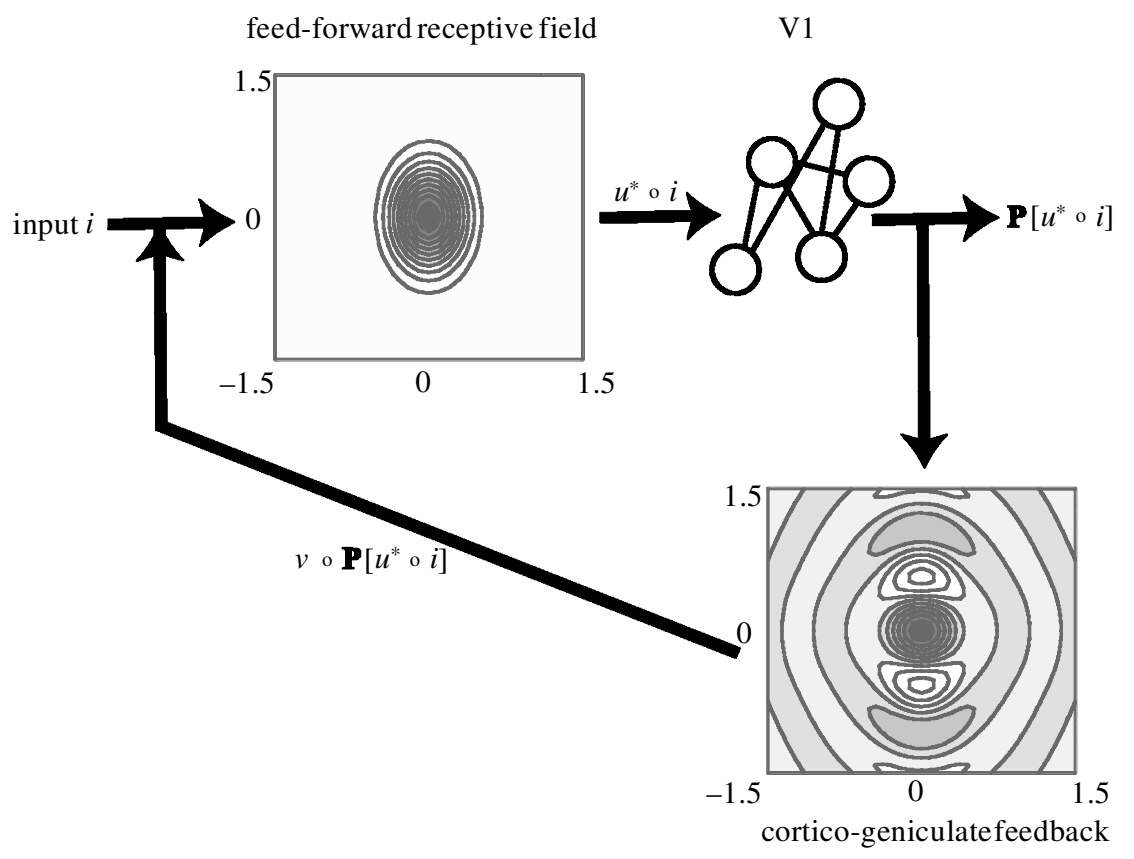

Figure 27. Schematic diagram of feed-forward, recurrent and feedback pathways that could be involved in the generation of a faithful representation of spatial frequency. The contour plot of a feed-forward (difference-of-Gaussians) receptive field profile is shown in retinal coordinates, together with the corresponding pattern of feedback connections. The latter is calculated using linear filter theory. The length scale is in units of the range of feed-forward excitation. Dark and light regions represent excitatory and inhibitory synapses, respectively.

and is a consistent finding in both simple and complex cells. Here, we present a possible cortical mechanism for cross-orientation suppression, based on the idea that the local circuits of a hypercolumn amplify the first spherical harmonic components of a stimulus. Consider a stimulus consisting of the sum of two gratings with identical spatial frequency $p_{\mathrm{s}}$ and distinct orientations $\Phi$ and $\Phi^{\prime}$, respectively:

$$
\begin{aligned}
i(\mathbf{r})= & \frac{C_{\mathrm{s}}}{2} \cos \left(p_{\mathrm{s}}[x \cos \Phi+y \sin \Phi)\right. \\
& +\frac{C_{\mathrm{s}}}{2} \cos \left(p_{\mathrm{s}}\left[x \cos \Phi^{\prime}+y \sin \Phi^{\prime}\right]\right) .
\end{aligned}
$$

When this stimulus is filtered by the receptive field (5.5), the resulting LGN input is (neglecting spatial phase)

$$
\begin{aligned}
h_{\mathrm{LGN}}= & \frac{C_{\mathrm{s}}}{2} \exp \left[-\frac{A^{2} p_{\mathrm{s}}^{2}}{2 p^{2}}\left(\kappa^{-2} \cos ^{2}(\Phi-\phi)+\sin ^{2}(\Phi-\phi)\right)\right] \\
& +\frac{C_{\mathrm{s}}}{2} \exp \left[-\frac{A^{2} p_{\mathrm{s}}^{2}}{2 p^{2}}\left(\kappa^{-2} \cos ^{2}\left(\Phi^{\prime}-\phi\right)+\sin ^{2}\left(\Phi^{\prime}-\phi\right)\right)\right] \\
& -C_{\mathrm{s}} \alpha \exp \left[-\frac{\hat{\kappa}^{2} A^{2} p_{\mathrm{s}}^{2}}{2 p^{2}}\right]=\frac{C_{\mathrm{s}}}{2} \sum_{n=0}^{\infty} U_{n}\left(p_{\mathrm{s}} \mid p\right) \\
& {\left[\cos 2 n(\Phi-\phi)+\cos 2 n\left(\Phi^{\prime}-\phi\right)\right], }
\end{aligned}
$$

where we have used equations (6.7) and (6.8). If we now project out the first-order spherical harmonic components, we obtain an effective LGN input of the form

$$
\begin{aligned}
h(\theta, \phi)= & C(1-\varepsilon)+C \varepsilon[\cos \Theta \cos \theta+\sin \Theta \\
& \left.\sin \theta \frac{\cos (2[\phi-\Phi])+\cos \left(2\left[\phi-\Phi^{\prime}\right]\right)}{2}\right],
\end{aligned}
$$

where $\Theta=\mathcal{L}\left(p_{\mathrm{s}}\right)$ (assuming some form of renormalization similar to that of $₫ 7$ ).
Suppose that $\Theta \neq 0, \pi$. If $\Phi^{\prime}=\Phi$, then we recover the case of a single grating with

$$
\begin{aligned}
h(\theta, \phi)= & C(1-\varepsilon)+C \varepsilon[\cos \Theta \cos \theta \\
& +\sin \Theta \sin \theta \cos (2[\phi-\Phi])],
\end{aligned}
$$

so that the peak cortical response is at $\theta=\Theta$ and $\phi=\Phi$. Conversely, in the case of an orthogonal grating, $\Phi^{\prime}=\Phi+\pi / 2$, there is exact cancellation of cosines such that

$h(\theta, \phi)=C(1-\varepsilon)+C \varepsilon \cos \Theta \cos \theta$

and the maximal cortical response will occur at either the low-frequency pinwheel $\theta=0$ (when $\cos \Theta>0$ ) or the high-frequency pinwheel $\theta=\pi$ (when $\cos \Theta<0$ ). More generally, we can rewrite equation (8.3) as

$$
\begin{aligned}
h(\theta, \phi)= & \bar{C}(1-\bar{\varepsilon})+\bar{\varepsilon} \bar{C}[\cos \bar{\Theta} \cos \theta \\
& +\sin \bar{\Theta} \sin \theta \cos (2[\phi-\bar{\Phi}])],
\end{aligned}
$$

where

$\bar{\Phi}=\frac{\Phi+\Phi^{\prime}}{2}$,

$\tan \bar{\Theta}=\cos \left(\Phi-\Phi^{\prime}\right) \tan \Theta$

and

$\bar{C}=C(1-\varepsilon+\varepsilon \kappa), \bar{\varepsilon}=\frac{\varepsilon \kappa}{1-\varepsilon+\varepsilon \kappa}$

with

$\kappa=\sqrt{\cos ^{2} \Theta+\sin ^{2} \Theta \cos ^{2}\left(\Phi-\Phi^{\prime}\right)}$.

The variation of $\bar{\Theta}, \bar{C}$ and $\bar{\varepsilon}$ with orientation difference $\Delta \Phi=\Phi-\Phi^{\prime}$ is shown in figures 28 and 29 . Two separate effects of cross-orientation suppression can be identified. 


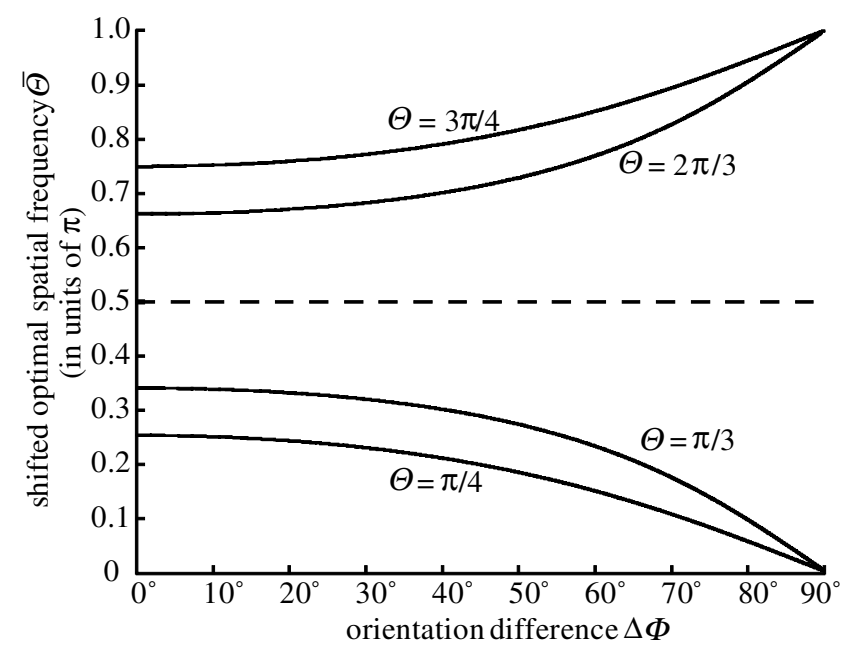

Figure 28. Cross-orientation suppression: shift in optimal spatial frequency of cortical response due to a pair of sinusoidal gratings with relative orientation $\Delta \Phi$ and the same spatial frequency $p_{\mathrm{s}}=\mathcal{L}^{-1}(\Theta)$. Here, the shifted spatial frequency $\bar{\Theta}$ is plotted as a function of $\Delta \Phi$ for various values of $\Theta$ and $\varepsilon=0.2$. Note that the peak is shifted to lower spatial frequencies when $0<\Theta<\pi / 2$ and higher spatial frequencies when $\pi / 2<\Theta<\pi$.

(a)

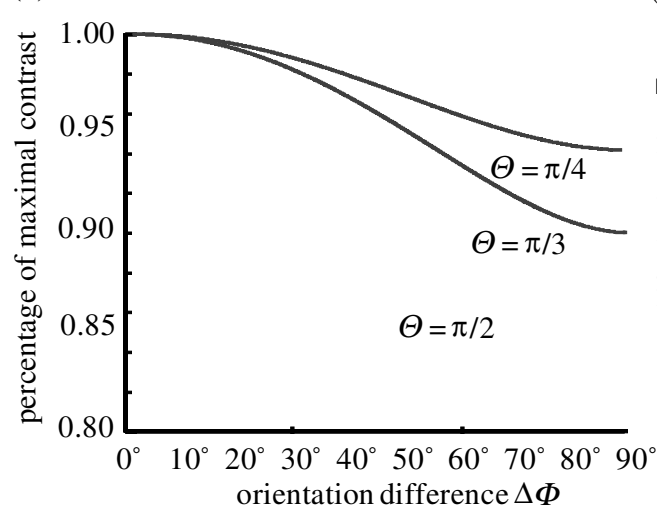

(b)

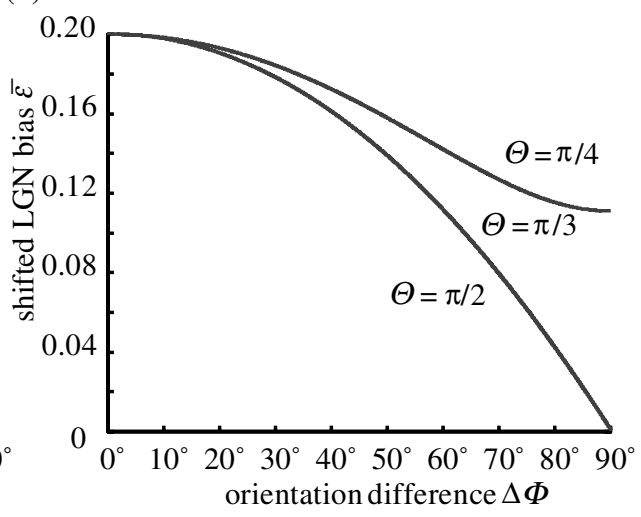

Figure 29. Cross-orientation suppression: reduction in LGN bias and contrast due to a pair of sinusoidal gratings with relative orientation $\Delta \Phi$ and the same spatial frequency $p_{\mathrm{s}}=\mathcal{2}^{-1}(\Theta)$. (a) Relative LGN contrast $\bar{C} / C$ is plotted as a function of $\Delta \Phi$ for various $\Theta$. (b) Shifted LGN bias $\bar{\varepsilon}$ is plotted as a function of $\Delta \Phi$ for various $\Theta$. Here, $\varepsilon=0.2$.

First, there is a shift in the peak response $\Theta \rightarrow \Theta^{\prime}$ and $\Phi \rightarrow \Phi^{\prime}$, which means that there will be local cortical suppression since cells that respond optimally at $(\Theta, \Phi)$ will have their response suppressed. However, other cells will have their response enhanced. Second, there is a reduction in both the effective contrast and bias of the LGN input, which implies that there is also global suppression due to a reduction in the cortical gain $G$ defined by equation (3.10). Both effects increase with $\Delta \Phi$, reaching a maximum when the gratings are orthogonal. On the other hand, the degree of suppression decreases as $\Theta$ approaches one of the pinwheels. (The point $\Theta=\pi / 2$ is a singular case, since there is no shift in spatial frequency with $\Delta \Phi$ but the LGN bias vanishes when $\Delta \Phi=\pi / 2$ ).

Cross-orientation suppression is also expected to occur for a checkerboard pattern, which is constructed by taking the product of two orthogonal sinusoidal gratings, one vertical and the other horizontal, say

$$
\begin{aligned}
i(\mathbf{r})= & C_{\mathrm{s}} \cos \left(p_{\mathrm{s}} x\right) \cos \left(p_{\mathrm{s}} y\right)=\frac{C_{\mathrm{s}}}{2} \cos \left(p_{\mathrm{s}}(x+y)\right) \\
& +\frac{C_{\mathrm{s}}}{2} \cos \left(p_{\mathrm{s}}(x-y)\right) .
\end{aligned}
$$

Such a stimulus decomposes as a sum of two gratings at $\pm 45^{\circ}$ and effective spatial frequency $\sqrt{2} p_{\mathrm{s}}$. Of course, it is possible to perceive checkerboards, crosses and other more complex stimuli. Hence, the occurrence of crossorientation suppression suggests that this is achieved at a more global level by combining the responses of many hypercolumns. It might also be possible that a hypercolumn amplifies higher-order harmonic components of a stimulus, and uses this to resolve certain aspects of composite stimuli. However, as discussed by Carandini \& Ringach (1997) within the context of the ring model, this could lead to the undesirable side effect of spurious peaks in the tuning curves.

\section{DISCUSSION}

The main conclusion of this paper is that orientation and spatial frequency can be represented by the surface coordinates of a sphere in each region of V1 that corresponds to a Hubel-Wiesel hypercolumn. We re-emphasize that this proposed spherical or $O(3)$ symmetry is an internal symmetry of the network topology, or equivalently, of the cortical labels for orientation and spatial- 
frequency preferences, and is not a symmetry of the actual spatial arrangement of neurons in a hypercolumn. Such a spherical coordinate system naturally accommodates the existence of orientation preference pinwheels and their association with regions of both low and high spatialfrequency preference. It follows that pattern formation on the sphere generated essentially by a symmetry breaking instability, in which the first few spherical harmonics are excited by incoming stimuli, can provide a mechanism for the existence of localized orientation and spatial frequency preferences and tuning, very much as suggested by De Valois \& De Valois (1988). A major consequence of the spherical topology and its association with orientation preference pinwheels is that orientation and spatialfrequency tuning curves are not separable.

The inclusion of spatial frequency preference and tuning as a property of V1 neurons is not as straightforward as in the case of orientation. If a local visual stimulus is filtered by the action of the geniculo-cortical pathway, as originally suggested by Hubel \& Wiesel (1962) for the orientation preference label, then the representation of spatial frequency is not faithful. Thus, figure 23 shows that the spatial frequency encoded by the hypercolumn in general differs from the actual spatial frequency of the stimulus. We conclude that to obtain a faithful representation of spatial frequency, we must insert another filtering operation. The most plausible and interesting possibility is that cortico-geniculate feedback generates such a filter. Thus, we propose a role for the back-projection from V1 to the LGN: it exists, in part, to provide a means to continually update and modify the LGN input to V1 so that the representation of spatial frequency remains faithful when signalled by the projection onto the first-order spherical harmonics. Interestingly, we find that the cortico-geniculate filter that results from our calculations is approximately the reciprocal of the feed-forward filter. Thus, it innervates the LGN in a pattern determined by the orientation and spatial frequency biases of the feedforward receptive field. We note that Murphy et al. (1999) found such patterns, at least in the case of orientation preferences. Recent observations by Sharon \& Grinvald (2002) also appear to be consistent with the model. They found that orientation tuning is amplified during a cortical evoked response. They also established that the time course of this amplification is not smooth but slows down ca. $50 \mathrm{~ms}$ after onset and then accelerates again. This is consistent with the postulated feedback process coming online after ca. $50 \mathrm{~ms}$.

Our suggestion regarding the function of the corticogeniculate feedback pathway differs considerably from many others. These other proposals have been concerned with such functions as gating retino-geniculate transmission, improving the precision of spike timing in LGN cells, enhancing the spatial-frequency tuning of LGN cells, and synchronizing slow oscillations between V1 and LGN (see Funke et al. 2001). Our model is closest in concept to that of Rao \& Ballard (1999) who suggested that in general, feedback connections carry predictions of lower level activities, whereas the corresponding feed-forward connections carry the residual errors between the predictions and the actual lower level activities. It remains to be determined what the connection is, if any, between our ideas and those concerning such predictive coding.
More specific results and predictions of our spherical model are as follows.

(i) Orientation preference and tuning should become weaker at low and high spatial frequencies, in part since, by hypothesis, such frequencies are located at the poles of the sphere. This is consistent with the early recordings of Hubel \& Wiesel (1962), who found numerous cells with poor or no tuning for orientation, many of which they later located in COblob regions of V1 (Livingstone \& Hubel 1984), now known to be regions of low spatial-frequency tuning (Hübener et al. 1997).

(ii) Spatial-frequency preference shifts occur at both ends of the frequency spectrum. The direction of the shifts is always towards the high- or low-frequency poles. Thus, low spatial frequencies tend to be signalled as even lower, high spatial frequencies as even higher. Issa et al. (2000) report such shifts at the low end of the spectrum. However, these authors also report high frequency shifts in the opposite direction to our predictions. We suggest that feedback could be responsible for the predicted change in direction of shifts in spatial-frequency preferences. That is, downward shifts are consistent with the properties of the geniculo-cortical pathways before the effects of the cortico-geniculate feedback have time to act. We predict that the earliest responses of cortical neurons should all exhibit downward shifts in spatial-frequency tuning, but for high spatial frequencies, such shifts should eventually disappear or even reverse, when measured later. Some observations appear to be consistent with this prediction (Bredfeldt \& Ringach 2002).

(iii) The contrast in variance of tuning curves for both orientation and spatial frequency is a natural property of our model, and indeed, of any model with an amplification mechanism. In this respect it differs somewhat from the quadratic threshold mechanism recently suggested by Miller \& Troy (2002), in that even the linear rectifier defined in equation (2.2) will generate contrast-invariant responses if there is an amplification process.

(iv) Finally, we remark that cross-orientation suppression is also a natural property of our model which also follows from the amplification process. However, the suppression mechanism is complicated. First, there is a local suppression effect-cells that respond optimally at $(\Theta, \Phi)$ will have their response suppressed by an orthogonal input. However, other cells will have their response enhanced. Second, there is also global suppression due to a reduction in the cortical gain $G$ defined by equation (3.10). Both effects increase with $\Delta \Phi$, reaching a maximum when the gratings are orthogonal. However, the degree of suppression decreases as $\Theta$ approaches one of the pinwheels.

We thank Professor Martin Golubitsky and Dr Peter Thomas for useful comments, Mr Michael Buice for mathematical proofreading and Miss Tanya Baker for help with figure 5. We also thank the referees for their thorough reading of the paper and their many helpful suggestions. This work was supported 
in part by grant 0209824 from the NSF to P.C.B. and by grant 96-24 from the James S. McDonnell Foundation to J.D.C.

\section{APPENDIX A}

We analysed the stability of the localized state (centred at the $\Theta=0$ pinwheel) by linearizing equation (3.4) about the fixed-point solution (equation (3.9)). First, we set

$I_{0}(t)=I_{0}+\gamma_{0}(t)$,

$I_{1}^{0}(t)=I_{1}+\gamma_{1}(t)$,

$I_{0}^{ \pm}(t)=\gamma_{ \pm}(t)$,

with $I_{0}$ and $I_{1}$ determined by the self-consistency conditions (3.30)-(3.32) and write

$\frac{\mathrm{d} a}{\mathrm{~d} t}=-a+\left[I_{0}+I_{1} \cos \theta+\gamma_{0}(t)+\sum_{m=1, \pm} \gamma_{m}(t) f_{m}(\theta, \phi)\right]_{+}$.

At a given time $t$, the boundary condition for the vanishing of the total synaptic drive is

$\mathcal{F}(\theta, \phi, t) \equiv I_{1} \cos \theta+\gamma_{0}(t)+\sum_{m} \gamma_{m}(t) f_{m}(\theta, \phi)=0$.

This equation can be linearized by setting $\theta=\theta_{c}$ $+\delta \theta(\phi, t)$ with

$\delta \theta(\phi, t)=\frac{\gamma_{0}(t)+\sum_{m=1, \pm} \gamma_{m}(t) f_{m}\left(\theta_{c}, \phi\right)}{I_{1} \sin \theta_{c}}$.

We have used the fact that $I_{0}+I_{1} \cos \theta_{\mathrm{c}}=0$. The next step is to take moments of equation (A 2) with respect to the zeroth and first order harmonics:

$\frac{\mathrm{d} R_{0}}{\mathrm{~d} t}=-R_{0}+\int_{0}^{\pi} \int_{0}^{\theta_{\mathrm{c}}+\delta \theta} \mathcal{H}(\theta, \phi, t) \mathscr{D}(\theta, \phi)$

and

$\frac{\mathrm{d} R_{1}^{n}}{\mathrm{~d} t}=-R_{1}^{n} \int_{0}^{\pi} \int_{0}^{\theta_{\mathrm{c}}+\delta \theta} f_{n}(\theta, \phi) \mathcal{H}(\theta, \phi, t) \mathscr{D}(\theta, \phi)$.

To linearize these equations set $R_{0}(t)=R_{0}+r_{0}(t)$, $R_{1}^{0}(t)=R_{1}+r_{1}(t)$ and $R_{1}^{ \pm}(t)=r_{ \pm}(t)$ with $R_{0}, R_{1}$ given by equations (3.31) and (3.32). It follows from equations (3.5), (3.6) and (3.30) that

$\gamma_{0}(t)=W_{0} r_{0}(t), \gamma_{m}(t)=W_{1} r_{m}(t)$,

for $m=1, \pm$. Here, $r_{0}(t)$ and $r_{1}(t)$ represent 'longtitudinal' fluctuations of the localized state whereas $r_{ \pm}(t)$ represent 'transverse' fluctuations. It transpires that the longitudinal and transverse fluctuations decouple at the linear level. Expanding equations (A 5) and (A 6) to first order in $\delta \theta$ we find that the longitudinal modes satisfy the pair of equations

$$
\begin{aligned}
\frac{\mathrm{d} r_{0}}{\mathrm{~d} t}= & -r_{0}+\frac{1}{4}\left(2 I_{0} \sin \theta_{\mathrm{c}}+I_{1} \sin 2 \theta_{\mathrm{c}}\right) \overline{\delta \theta} \\
& +\frac{\gamma_{0}\left[1-\cos \theta_{\mathrm{c}}\right]}{2}+\frac{\gamma_{1}\left[1-\cos 2 \theta_{\mathrm{c}}\right]}{8}
\end{aligned}
$$

and

$$
\begin{aligned}
\frac{\mathrm{d} r_{1}}{\mathrm{~d} t}= & -r_{1}+\frac{1}{4}\left(I_{0} \sin 2 \theta_{\mathrm{c}}+2 I_{1} \cos ^{2} \theta_{\mathrm{c}} \sin \theta_{\mathrm{c}}\right) \overline{\delta \theta} \\
& +\frac{\gamma_{0}\left[1-\cos 2 \theta_{\mathrm{c}}\right]}{8}+\frac{\gamma_{1}\left[1-\cos ^{3} \theta_{\mathrm{c}}\right]}{6},
\end{aligned}
$$

where

$\overline{\delta \theta}=\frac{1}{\pi} \int_{0}^{\pi} \delta \theta(\phi) d \phi=\frac{\gamma_{0}+\gamma_{1} \cos \theta_{c}}{I_{1} \sin \theta_{c}}$.

Equation (3.29) then implies that the coefficients multiplying $\overline{\delta \theta}$ actually vanish. Thus, we have the simple matrix equation

$\frac{\mathrm{d}}{\mathrm{d} t}\left(\begin{array}{l}r_{0} \\ r_{1}\end{array}\right)=\mathbf{W}\left(\theta_{\mathrm{c}}\right)\left(\begin{array}{l}r_{0} \\ r_{1}\end{array}\right)$,

where

$\mathbf{W}\left(\theta_{\mathrm{c}}\right)=\left(\begin{array}{cc}-1+\frac{W_{0}\left[1-\cos \theta_{c}\right]}{2} & \frac{W_{1}\left[1-\cos 2 \theta_{c}\right]}{8} \\ \frac{W_{0}\left[1-\cos 2 \theta_{c}\right]}{8} & -1+\frac{W_{1}\left[1-\cos ^{3} \theta_{c}\right]}{6}\end{array}\right)$.

Hence, we obtain an eigenvalue equation of the form

$\lambda^{2}-\lambda \operatorname{Tr} \mathbf{W}\left(\theta_{\mathrm{c}}\right)+\operatorname{det} \mathbf{W}\left(\theta_{\mathrm{c}}\right)=0$.

One can show that the localized state undergoes amplitude instability as $W_{0}$ is increased for fixed $W_{1}$ due to a single real eigenvalue becoming positive. The condition for such instability is $\operatorname{det} \mathbf{W}\left(\theta_{\mathrm{c}}\right)=0$. In the particular case of a homogeneous input $(\varepsilon=0)$, one finds from equations (3.26) and (3.27) that

$\operatorname{det} \mathbf{W}\left(\theta_{\mathrm{c}}\right)=-\frac{W_{1}}{8}\left[1-\cos 2 \theta_{\mathrm{c}}\right]\left[\cos \theta_{\mathrm{c}}+W_{0} A_{0}\left(\theta_{\mathrm{c}}\right)\right]$,

so that the condition for an amplitude instability is

$W_{1}=W_{\mathrm{c}} \equiv-\frac{\cos \theta_{\mathrm{c}}}{A_{0}\left(\theta_{\mathrm{c}}\right)}$.

Finally, expanding equation (A 6) to first order in $\delta \theta$ for the transverse modes, we find that

$$
\begin{aligned}
\frac{\mathrm{d} r_{ \pm}}{\mathrm{d} t}= & -r_{ \pm}+\frac{1}{2}\left(I_{0} \sin ^{2} \theta_{\mathrm{c}}+I_{1} \sin ^{2} \theta_{\mathrm{c}} \cos \theta_{\mathrm{c}}\right){\overline{\delta \theta_{ \pm}}} \\
& +\gamma_{ \pm} W_{1} \mathcal{A}_{1}\left(\theta_{\mathrm{c}}\right) r_{ \pm}
\end{aligned}
$$

where

$\overline{\delta \theta}_{ \pm} \equiv \frac{1}{\pi} \int_{0}^{\pi}\left(\begin{array}{c}\cos \theta_{\mathrm{c}} \\ \sin \theta_{\mathrm{c}}\end{array}\right) \delta \theta(\phi) \mathrm{d} \phi=\frac{W_{1} r_{ \pm}}{2 I_{1}}$.

Equation (3.29) implies that the coefficient multiplying vanishes $\overline{\delta \theta}_{ \pm}$. Hence,

$\frac{\mathrm{d} r_{ \pm}}{\mathrm{d} t}=\left[-1+W_{1} \mathcal{A}_{1}\left(\theta_{\mathrm{c}}\right)\right] r_{ \pm}$.

It immediately follows that in the case of a homogeneous input, the localized state is marginally stable with respect to excitation of the transverse modes since $1=W_{1} \mathcal{l}_{1}\left(\theta_{\mathrm{c}}\right)$.

\section{APPENDIX B}

To simplify our analysis of the spherical model, we collapsed the inhibitory and excitatory cell populations into a single equivalent population. Such a simplification greatly reduces the number of free parameters of the system. 
However, the basic insights gained from the one-population model can now be used to develop the mean field theory of a more realistic two-population model.

Let us denote the activity of the excitatory (e) and inhibitory $(i)$ populations by $a_{r}(\theta, \phi, t)$ with $r=e, i$. A twopopulation version of equation (2.2) is then

$\frac{\partial a_{r}(\theta, \phi, t)}{\partial t}=-a_{r}(\theta, \phi, t)+\beta_{r}\left[I_{r}(\theta, \phi, t)-\kappa_{r}\right]_{+}$,

where $\kappa_{r}$ is the threshold and $I_{r}(\theta, \phi, t)$ is the total synaptic current of the $r$ th population,

$$
\begin{aligned}
I_{r}(\theta, \phi, t)= & h_{r}(\theta, \phi) \\
& +\sum_{s=e, \mathrm{i}} \int_{s^{2}} w_{r s}\left(\theta, \phi \mid \theta^{\prime}, \phi^{\prime}\right) a_{s}\left(\theta^{\prime}, \phi^{\prime}, t\right) \mathscr{D}\left(\theta^{\prime}, \phi^{\prime}\right) .
\end{aligned}
$$

We have also introduced input gains $\beta_{r}$. The weight distributions $w_{r s}$ connecting the various cell populations are taken to be $O(3)$ invariant, and are constructed out of zeroth and first-order spherical harmonics according to

$$
\begin{aligned}
w_{e e}\left(\theta, \phi \mid \theta^{\prime}, \phi^{\prime}\right)= & w_{i e}\left(\theta, \phi \mid \theta^{\prime}, \phi^{\prime}\right)=W_{e, 0} \\
& +W_{e, 1} \sum_{m=0, \pm} f_{m}(\theta, \phi) f_{m}\left(\theta^{\prime}, \phi^{\prime}\right)
\end{aligned}
$$

and

$$
\begin{aligned}
w_{e i}\left(\theta, \phi \mid \theta^{\prime}, \phi^{\prime}\right)= & w_{i i}\left(\theta, \phi \mid \theta^{\prime}, \phi^{\prime}\right)=-W_{i, 0} \\
& -W_{i, 1} \sum_{m=0, \pm} f_{m}(\theta, \phi) f_{m}\left(\theta^{\prime}, \phi^{\prime}\right)
\end{aligned}
$$

with $W_{r, 1}, W_{r, 0} \geqslant 0$. As in the one-population model, the weakly biased LGN input $h_{r}(\theta, \phi)$ is assumed to be of the form

$$
h_{r}(\theta, \phi)=\alpha_{r} h(\theta, \phi) \text {, }
$$

with $h$ given by equation (2.10) and $\alpha_{r}$ determining the relative strength of the input to the two populations. Introducing the order parameters

$R_{r, 0}(t)=\int_{s^{2}} a_{r}(\theta, \phi, t) \mathscr{D}(\theta, \phi)$,

$R_{r, 1}^{m}(t)=\int_{s^{2}} a_{r}(\theta, \phi, t) f_{m}(\theta, \phi) \mathscr{D}(\theta, \phi)$,

equation (B 1) can be rewritten in the form

$\frac{\partial a_{r}(\theta, \phi, t)}{\partial t}=-a_{r}(\theta, \phi, t)+\left[I_{r, 0}(t)+\sum_{m=0, \pm} I_{r, 1}^{m}(t) f_{m}(\theta, \phi)\right]_{+}$,

where

$I_{r, 0}=\beta_{r}\left[\alpha_{r} C(1-\varepsilon)-\kappa+W_{e, 0} R_{e, 0}-W_{i, 0} R_{i, 0}\right]$

and

$I_{r, 1}^{m}=\beta_{r}\left[\alpha_{r} C \varepsilon f_{m}(\Theta, \Phi)+W_{e, 1} R_{e, 1}^{m}-W_{i, 1} R_{i, 1}^{m}\right]$.

We focus on a fixed-point solution of equation (B 8) in which both the excitatory and inhibitory populations are in a stationary localized state of the form

$a_{r}(\theta, \phi)=I_{r, 1}\left[\sum_{m=0, \pm} f_{m}(\Theta, \Phi) f_{m}(\theta, \phi)-\cos \theta_{c, r}\right]_{+}$.

Taking moments of equation (B 8) with respect to the zeroth and first-order spherical harmonics and proceeding along identical lines to the one-population model (see $₫ 3$ ), one finds that such a solution exists provided that

$R_{r, 1}^{m}=R_{r, 1} f_{m}(\Theta, \Phi), \quad I_{r, 1}^{m}=I_{r, 1} f_{m}(\Theta, \Phi)$,

with

$R_{r, 0}=I_{r, 1} \mathcal{A}_{0}\left(\theta_{c, r}\right), R_{r, 1}=I_{r, 1} \mathcal{A}_{1}\left(\theta_{c, r}\right)$

and

$I_{r, 0}+I_{r, 1} \cos \theta_{c, r}=0$.

The functions $\mathscr{A}_{0}, \mathscr{A}_{1}$ are defined by equations (3.26) and (3.27). Combining equations (B 11) and (B 12) with equations (B 8) and (B 9) leads to the mean-field equations

$R_{r, 0}=\beta_{r}\left[\alpha_{r} C \varepsilon+W_{e, 1} R_{e, 1}-W_{i, 1} R_{i, 1}\right] \mathcal{C}_{0}\left(\theta_{c, r}\right)$

and

$R_{r, 1}=\beta_{r}\left[\alpha_{r} C \varepsilon+W_{e, 1} R_{e, 1}-W_{i, 1} R_{i, 1}\right] \mathcal{l}_{1}\left(\theta_{c, r}\right)$.

(a) Marginal phase $(\varepsilon=0)$

In the case of a homogeneous input, equation (B 15) reduces to the matrix equation

$\mathbf{W}\left(\theta_{c, e}, \theta_{c, i}\right)\left(\begin{array}{c}R_{e, 1} \\ R_{i, 1}\end{array}\right)=0$,

where

$\mathbf{W}\left(\theta_{c, e} \theta_{c, i}\right)=\left(\begin{array}{cc}-1+\beta_{e} W_{e, 1} \mathcal{L}_{1}\left(\theta_{c, e}\right) & -\beta_{e} W_{i, 1} \mathcal{L}_{1}\left(\theta_{c, e}\right) \\ \beta_{i} W_{e, 1} \mathcal{L}_{1}\left(\theta_{c, i}\right) & -1-\beta_{i} W_{i, 1} \mathcal{L}_{1}\left(\theta_{c, i}\right)\end{array}\right)$.

A necessary condition for the existence of a non-trivial localized state can then be expressed as $\operatorname{det} \mathbf{W}\left(\theta_{c, e}, \theta_{c, i}\right)$ $=0$, that is,

$\beta_{e} W_{e, 1} \mathcal{A}_{1}\left(\theta_{c, e}\right)-\beta_{i} W_{i, 1} \mathcal{A}_{1}\left(\theta_{c, i}\right)=1$.

\section{REFERENCES}

Angelucci, A., Levitt, J. B. \& Lund, J. S. 2001 Anatomical origins of the classical receptive field and modulatory surround field of single neurons in macaque visual cortical area V1. Progr. Brain Res. 136, 373-384.

Arfken, G. 1985 Mathematical methods for physicists, 3rd edn. San Diego: Academic Press.

Ben-Yishai, R., Bar-Or, R. L. \& Sompolinsky, H. 1995 Theory of orientation tuning in visual cortex. Proc. Natl Acad. Sci. USA 92, 3844-3848.

Ben-Yishai, R., Hansel, D. \& Sompolinsky, H. 1997 Traveling waves and the processing of weakly tuned inputs in a cortical network module. F. Comput. Neurosci. 4, 57-77.

Blasdel, G. G. 1992 Orientation selectivity, preference, and continuity in monkey striate cortex. F. Neurosci. 12, 31393161.

Blasdel, G. G. \& Salama, G. 1986 Voltage-sensitive dyes reveal a modular organization in monkey striate cortex. Nature 321, 579-585.

Bonhoeffer, T. \& Grinvald, A. 1991 Iso-orientation domains in cat are organized in pinwheel-like patterns. Nature 353, 429-431.

Bonhoeffer, T., Kim, D. S., Malonek, D., Shoham, D. \& Grinvald, A. 1995 Optical imaging of the layout of functional domains in area $17 / 18$ border in cat visual cortex. Eur. $\mathcal{F}$. Neurosci. 7, 1973-1988. 
Bredfeldt, C. E. \& Ringach, D. L. 2002 Dynamics of spatial frequency tuning in macaque V1. F. Neurosci. 22, 19761984.

Bressloff, P. C. \& Cowan, J. D. $2002 a$ An amplitude equation approach to contextual effects in primary visual cortex. Neural Comput. 14, 493-525.

Bressloff, P. C. \& Cowan, J. D. $2002 b$ An SO(3) symmetry breaking mechanism for orientation and spatial-frequency tuning in visual cortex. Phys. Rev. Lett. 88, 078102.

Bressloff, P. C., Bressloff, N. W. \& Cowan, J. D. 2000 Dynamical mechanism for sharp orientation tuning in an integrate-and-fire model of a cortical hypercolumn. Neural Comput. 12, 2473-2511.

Carandini, M. \& Ringach, D. 1997 Predictions of a recurrent model of orientation selectivity. Vis. Res. 37, 3061-3071.

DeAngelis, G. C., Robson, J. G., Ohzawa, I. \& Freeman, R. D. 1992 Organization of suppression in receptive fields of neurons in cat visual cortex. F. Neurophysiol. 68, 144-163.

De Valois, R. L. \& De Valois, K. K. 1988 Spatial vision. Oxford University Press.

De Valois, R. L., Albrecht, D. G. \& Thorell, L. G. 1982 Spatial frequency selectivity of cells in macaque visual cortex. Vis. Res. 22, 545-559.

Douglas, R. J., Koch, C., Mahowald, M., Martin, K. A. C. \& Suarez, H. H. 1995 Recurrent excitation in neocortical circuits. Science 269, 981-985.

Dragoi, V. \& Sur, M. 2000 Some properties of recurrent inhibition in primary visual cortex: contrast and orientation dependence on contextual effects. F. Neurophysiol. 83, 1019-1030.

Ferster, D., Chung, S. \& Wheat, H. 1997 Orientation selectivity of thalamic input to simple cells of cat visual cortex. Nature 380, 249-281.

Funke, K., Kisvárdy, Z. F., Volgushev, M. \& Wörgötter, F. 2001 Integrating anatomy and physiology of the primary visual pathway: from LGL to cortex. In Models of neural networks IV, early vision and attention (ed. J. L. van Hemmen, J. D. Cowan \& E. Domany), pp. 97-182. New York: Springer.

Guillery, R. W., Feig, S. L. \& van Lieshout, D. P. 2001 Connections of higher order visual relays in the thalamus: a study of corticothalamic pathways in cats. F. Comp. Neurol. 438, $66-85$.

Hansel, D. \& Sompolinsky, H. 1997 Modeling feature selectivity in local cortical circuits. In Methods of neuronal modeling, 2nd edn (ed. C. Koch \& I. Segev), pp. 499-567. Cambridge, MA: MIT Press.

Hawken, M. J. \& Parker, A. J. 1987 Spatial properties of neurons in the monkey striate cortex. Proc. R. Soc. Lond. B 231, 251-288.

Horton, J. C. \& Hubel, D. H. 1981 Regular patchy distribution of cytochrome oxidase staining in primary visual cortex of macaque monkey. Nature 292, 762-764.

Hubel, D. H. \& Wiesel, T. N. 1962 Receptive fields, binocular interaction and functional architecture in the cat's visual cortex. F. Neurosci. 3, 1116-1133.

Hubel, D. H. \& Wiesel, T. N. 1968 Receptive fields and functional architecture of monkey striate cortex. F. Physiol. Lond. 195, 215-243.

Hubel, D. H. \& Wiesel, T. N. 1974 Uniformity of monkey striate cortex: a parallel relationship between field size, scatter, and magnification factor. F. Comp. Neurol. 158, 295-306.

Hubel, D. H. \& Wiesel, T. N. 1977 Functional architecture of macaque monkey visual cortex. Proc. R. Soc. Lond. B 198, $1-59$.

Hubel, D. H., Wiesel, T. N. \& Stryker, M. P. 1978 Anatomical demonstration of orientation columns in macaque monkey. f. Comp. Neurol. 177, 361-380.
Hübener, M., Shoham, D., Grinvald, A. \& Bonhoeffer, T. 1997 Spatial relationships among three columnar systems in cat area 17. F. Neurosci. 17, 9270-9284.

Issa, N. P., Trepel, C. \& Stryker, M. P. 2000 Spatial frequency maps in cat visual cortex. F. Neurosci. 20, 8504-8514.

Jones, J. P. \& Palmer, L. A. 1987 An evaluation of the twodimensional Gabor filter model of simple receptive fields in cat striate cortex. F. Neurophysiol. 58, 1233-1258.

Kelly, D. H. \& Magnuski, H. S. 1975 Pattern detection and the two-dimensional Fourier transform: circular targets. Vis. Res. 15, 911-915.

LeVay, S. \& Nelson, S. B. 1991 Columnar organization of the visual cortex. In The neural basis of visual function (ed. A. G. Leventhal), pp. 266-315. Boca Raton, FL: CRC Press.

Li, Z. 1999 Pre-attentive segmentation in the primary visual cortex. Spat. Vis. 13, 25-39.

Livingstone, M. S. \& Hubel, D. H. 1984 Anatomy and physiology of a color system in the primate visual cortex. F. Neurosci. 4, 309-356.

McLaughlin, D., Shapley, R., Shelley, M. \& Wielaard, D. J. 2000 A neuronal network model of macaque primary visual cortex (V1): orientation tuning and dynamics in the input layer 4C $\alpha$. Proc. Natl Acad. Sci. USA 97, 8087-8092.

Maldonado, P. E., Gödecke, I., Gray, C. M. \& Bonhoeffer, T. 1997 Orientation selectivity in pinwheel centers in cat striate cortex. Science 276, 1551-1555.

Mazer, J. A., Vinje, W. E., McDermott, J., Schiller, P. H. \& Gallant, J. L. 2002 Spatial frequency and orientation tuning dynamics in V1. Proc. Natl Acad. Sci. USA 99, 1645-1650.

Miller, K. D. \& Troy, T.W. 2002 Neural noise can explain expansive, power-law nonlinearities in neural response functions. F. Neurophysiol. 87, 653-659.

Mundel, T., Dimitrov, A. \& Cowan, J. D. 1997 Visual cortex circuitry and orientation tuning. In Advances in neural information processing systems, vol. 9 (ed. M. C. Mozer, M. I. Jordan \& T. Petsche), pp. 886-893. Cambridge, MA: MTT Press.

Murphy, K., Jones, D. G. \& Sluyters, R. C. V. 1995 Cytochrome oxidase blobs in cat primary visual cortex. $\mathcal{F}$. Neurosci. 15, 4196-4208.

Murphy, P. C., Duckett, S. G. \& Sillito, A. M. 1999 Feedback connections to the lateral geniculate nucleus and cortical response properties. Science 286, 1552-1554.

Nelson, S., Toth, L., Seth, B. \& Sur, M. 1994 Orientation selectivity of cortical neurons during extra-cellular blockade of inhibition. Science 265, 774-777.

Obermayer, K. \& Blasdel, G. 1993 Geometry of orientation and ocular dominance columns in monkey striate cortex. $\mathcal{F}$. Neurosci. 13, 4114-4129.

O’Keefe, L. P., Levitt, J. B., Kiper, D. C., Shapley, R. M. \& Movshon, J. A. 1998 Functional organization of owl monkey lateral geniculate nucleus and visual cortex. F. Neurophysiol. 80, 594-609.

Rao, R. P. N. \& Ballard, D. H. 1999 Predictive coding in the visual cortex: a functional interpretation of some extra-classical receptive-field effects. Nature Neurosci. 2, 79-87.

Reid, R. C. \& Alonso, J. M. 1995 Specificity of monosynaptic connections from thalamus to visual cortex. Nature 378, 281-284.

Roerig, B. \& Chen, B. 2002 Relationships of local inhibitory and excitatory circuits to orientation preference maps in ferret visual cortex. Cerebral Cortex 12, 187-198.

Sharon, D. \& Grinvald, A. 2002 Dynamics and constancy in cortical spatiotemporal patterns of orientation processing. Science 295, 512-515.

Sillito, A. M. 1975 The contribution of inhibitory mechanisms to the receptive-field properties of neurones in the striate cortex of the cat. F. Physiol. Lond. 250, 305-329. 
Somers, D. C., Nelson, S. \& Sur, M. 1995 An emergent model of orientation selectivity in cat visual cortical simple cells. $\mathcal{F}$. Neurosci. 15, 5448-5465.

Somers, D. C., Todorov, E. V., Siapas, A. G., Toth, L. J., Kim, D.-S. \& Sur, M. 1998 A local circuit approach to understanding integration of long-range inputs in primary visual cortex. Cerebral Cortex 8, 204-217.

Stetter, M., Bartsch, H. \& Obermayer, K. 2000 A mean field model for orientation tuning, contrast saturation, and contextual effects in the primary visual cortex. Biol. Cybernet. 87, 291-304.

Swindale, N. V. 1996 The development of topography in visual cortex: a review of models. Network 7, 161-247.
Tootell, R. B. H., Silverman, M. S. \& De Valois, R. L. 1981 Spatial frequency columns in primary visual cortex. Science 214, 813-815.

Vidyasagar, T. R., Pei, X. \& Volgushev, M. 1996 Multiple mechanisms underlying the orientation selectivity of visual cortical neurons. Trends Neurosci. 19, 272-277.

Webster, M. A. \& De Valois, R. L. 1985 Relationship between spatial-frequency and orientation tuning of striate-cortex cells. F. Opt. Soc. Am. A 2, 1124-1132.

Wiesel, T. N., Hubel, D. H. \& Lam, D. M. K. 1974 Autoradiographic demonstration of ocular-dominance columns in the monkey striate cortex by means of transneuronal transport. Brain Res. 79, 273-279. 